\title{
Graphene: Nanostructure engineering and applications
}

\author{
Tingting Zhang ${ }^{1,2}$, Shuang Wu $\mathbf{W u}^{1,2}$, Rong Yang ${ }^{1,2,3, *}$, Guangyu Zhang ${ }^{1,2,3,4, \dagger}$ \\ ${ }^{1}$ Institute of Physics, Chinese Academy of Sciences, Beijing 100190, China \\ ${ }^{2}$ School of Physical Sciences, University of Chinese Academy of Sciences, Beijing 100190, China \\ ${ }^{3}$ Beijing Key Laboratory for Nanomaterials and Nanodevices, Beijing 100190, China \\ ${ }^{4}$ Collaborative Innovation Center of Quantum Matter, Beijing 100190, China \\ Corresponding authors. E-mail: ${ }^{\dagger}$ gyzhang@iphy.ac.cn, ${ }^{*}$ ryang@iphy.ac.cn \\ Received December 8, 2016; accepted December 26, 2016
}

\begin{abstract}
Graphene has attracted extensive research interest in recent years because of its fascinating physical properties and its potential for various applications. The band structure or electronic properties of graphene are very sensitive to its geometry, size, and edge structures, especially when the size of graphene is below the quantum confinement limit. Graphene nanoribbons (GNRs) can be used as a model system to investigate such structure-sensitive parameters. In this review, we examine the fabrication of GNRs via both top-down and bottom-up approaches. The edge-related electronic and transport properties of GNRs are also discussed.
\end{abstract}

Keywords graphene nanoribbons (GNRs), microfabrication, top-down, bottom-up, electronic transport, zigzag, mobility, edge state

PACS numbers 81.07.-b, 73.22.Pr, 72.80.Vp, 72.20.Ee

Contents

1 Introduction

2 Nanostructure engineering of graphene

2.1 Top-down approaches

2.1.1 Oxygen-assisted lithography with different masks

2.1.2 Hydrogen-assisted etching without masks

2.1.3 Scanning probe lithography

2.1.4 Unzipping of carbon nanotubes

2.2 Bottom-up approaches

2.2.1 Self-assembly of organic molecules

2.2.2 Growth of graphene nanoribbons on templated substrates

2.3 Challenges in electronic-grade graphene nanoribbon processing

3 Electronic band structure of graphene nanoribbons

3.1 Differences between the band structures of zigzag and armchair nanoribbons

3.1.1 Edge state in zigzag graphene nanoribbons

3.1.2 Energy gap engineering in graphene nanoribbons
3.2 Scanning tunneling microscopy and spectroscopy observations of edge states and band gaps

3.2.1 Zigzag edge states 13

3.2.2 Energy gaps 14

4 Electron transport in graphene nanoribbons 15

24.1 Transport in graphene nanoribbons with different edges

4.1.1 Transport in graphene nanoribbons with rough edges

4.1.2 Transport in graphene nanoribbons with regular edges

4.2 Quantum Hall effect of two-terminal graphene nanoribbon devices

5 Conclusions and outlook

Acknowledgements

References

101 Introduction

10 Graphene, which has a one-atom-thick honeycomb carbon lattice, is a typical two-dimensional Dirac material

11 that has been widely explored since its discovery [1]. Owing to its opposite chiralities and a Berry phase of imet

12 the Dirac points, graphene is considered to be a fasci- 
nating system for studying physical phenomena in condensed matter physics, such as the half-integer quantum Hall effect [2], Klein tunneling [3], and ballistic transport $[4,5]$. Graphene is also recognized as one of the most promising candidates for future applications in nanoelectronics owing to its high carrier mobility [6].

Despite its high carrier mobility [6] and numerous extraordinary properties for electronic applications, one of the biggest challenges in utilizing graphene in nanoelectronics is the lack of a sizable band gap [2]. The absence of a band gap makes it difficult to switch off a graphene field effect transistor (FET), leading to a rather small on/off ratio. One solution to this problem is to engineer graphene into nanostructures. In particular, a sizable energy gap can be achieved in graphene by patterning it as graphene nanoribbons (GNRs) [7-9], nanomesh (GNM) $[10,11]$, or quantum dots (GQDs) [12]. GNRs are the most widely studied graphene nanostructure. When the size of the material becomes equivalent to the length that characterizes electron motion in the material, the properties of the material depend strongly on its size and shape. Owing to the quantum confinement induced by the GNR structure, a sizable band gap can be introduced, making it possible to use GNRs in nanoeletcronics. GNRs can gradually transform from semimetals to semiconductors, as the energy gap is inversely proportional to the width [7]. Moreover, GNRs can be categorized as zigzag GNRs (ZGNRs), armchair GNRs (AGNRs), or random edge GNRs based on their edge configurations (Fig. 1). Random edge GNRs can be characterized as combination of zigzag and armchair sites. As the electronic and transport properties of GNRs are greatly edge-dependence, exploring the electronic and transport properties of GNRs based on different edge geometries is of intense research interest.

Herein, we present an overview of recent work on GNR structure engineering and an exploration of their electronic and transport properties. We particularly focus on engineering the edge termination of GNRs and the resulting properties from both theoretical and experimental points of view. First, we briefly review the engineering of GNRs based on top-down and bottom-up approaches in Section 2. Then, in Sections 3 and 4, we focus on the electronic properties and transport properties of GNRs, respectively.

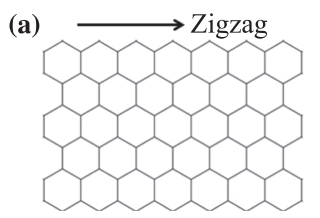

(b)

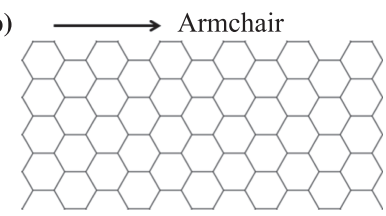

Fig. 1 (a) Zigzag graphene nanoribbon. (b) Armchair graphene nanoribbon.

\section{Nanostructure engineering of graphene}

Various synthesis routes in view of either top-down or bottom-up point have been established to engineer graphene into nanostructures.

\subsection{Top-down approaches}

\subsubsection{Oxygen-assisted lithography with different masks}

The most widely employed method for preparing GNRs is based on a top-down approach that utilizes lithography in combination with plasma etching processes. Various types of lithographic methods, such as electron beam lithography (EBL) [13] and nanosphere [14, 15], block copolymer (BCP) $[10,16,17]$, and nanowire [18-22] templated lithography, have been demonstrated for mask patterning, while oxygen plasma generated in a reactive ion etching (RIE) system is usually used to remove unmasked areas, leaving narrow stripes of graphene.

EBL plus oxygen plasma etching is the most widely adopted approach for the fabrication of graphene nanostructures [7]. Although precise EBL can give a line width of less than $10 \mathrm{~nm}$, the narrowest GNRs fabricated using this approach is $\sim 20 \mathrm{~nm}$ owing to the violent nature of oxygen plasma etching. As-fabricated GNRs are likely to be passivated by $\mathrm{O}-$ or $\mathrm{OH}-$ at the edges after etching, which will degrade the electronic properties. Moreover, the edge orientation cannot be controlled using this approach. An alternative lithographic method employing self-assembled BCPs as an etch mask has been developed to make GNM without the EBL process. In 2010, Bai et al. utilized a $\mathrm{BCP}(\mathrm{P}(\mathrm{S}-b-\mathrm{MMA}))$ as an etching template, followed by $\mathrm{CHF}_{3}$-based RIE to form a nanomesh hard mask. Subsequently, the unprotected graphene was etched using oxygen plasma, as shown in Fig. 2 [10]. A neck width of $\sim 5 \mathrm{~nm}$ could be fabricated by choosing appropriate molecular weights of $\mathrm{P}(\mathrm{S}-b-\mathrm{MMA})$ and aggressive over-etching conditions. GNM can sustain currents that are approximately 100 times larger than those tolerated by a single GNR device at room temperature, while the on/off ratio of GNM is comparable to that of a GNR. However, controlling the ultra-narrow neck width without breaking the interconnected GNM network is a challenge owing to the standard deviation of the harsh over-etching process, and this uncertainty may be much more stringent in larger GNM devices. Soon after, Kim et al. fabricated GNM with sub-20 nm features across $>1 \mathrm{~mm}^{2}$ using a similar BCP lithography method [16], indicating the potential of the $\mathrm{BCP}$ approach for fabricating graphene nanostructures on a large scale.

Compared with EBL technology, the BCP approach can realize both high resolution and scalability with low cost [23]. However, similar to the EBL and oxygen 
(a)

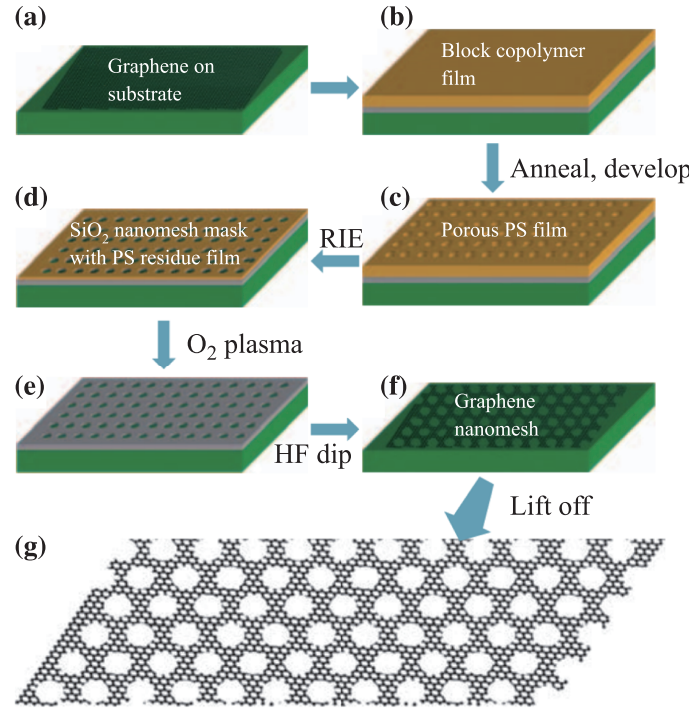

(i)

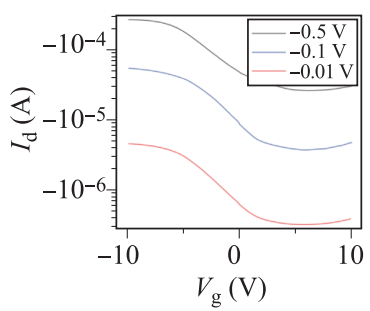

(h)

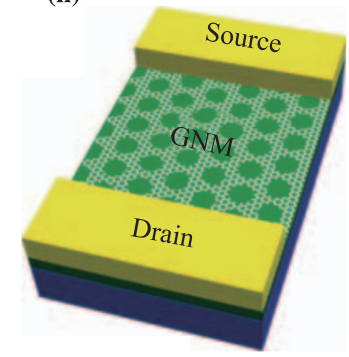

(j)

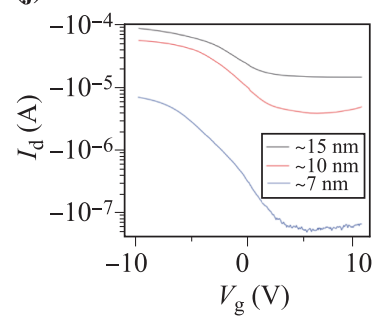

Fig. 2 Illustration of fabrication of graphene nanomesh (GNM) using BCP lithography. (a-g) The procedure of BCP lithography to form GNM. (h-j) Electrical properties of a graphene nanomesh device. Reproduced from Ref. [10].

plasma etching fabrication technique, graphene nanostructures fabricated using BCP lithography also have poor electrical performance. This rather low quality, caused by the doping effect and impurities, makes GNRs fabricated by BCP lithography less favorable for electrical transport studies and FETs. Another approach for obtaining GNRs is to use nanowires as an etch mask. Nanowires with smooth edges and widths of 1-2 $\mathrm{nm}$ can be synthesized using various chemical vapor approaches, which have shown promise for obtaining GNRs down to a few nanometers in width [24-27]. Duan's group fabricated GNRs with sub-10 nm width by utilizing silicon nanowires as an etch mask in oxygen plasma etching [28]. The resulting width of GNRs depends on the diameter of the silicon nanowire and can also be tuned by the oxygen plasma etching time. Interestingly, if oxide nanowires are chosen as masks [19-21], the nanowires can be directly used as a top gate in GNR device fabrication. This group has also used $\mathrm{Si} / \mathrm{HfO}_{2}$ core-shell nanowires as a mask to fabricate GNR FETs, as illustrated in Fig. 3 [19]. Using this method, highperformance FETs with the core-shell nanowires as a top gate have been obtained, with transconductance of $\sim 3.2 \mathrm{mS} \cdot \mu \mathrm{S}^{-1}$. This method reveals a new strategy for obtaining high-performance top-gate GNRs, which can integrate GNRs with a dielectric of controllable thickness.

The methods mentioned above can produce GNRs with widths down to a few nanometers and as a result, sizable band gaps can be opened owing to quantum confinement. However, it is still difficult to define the edge structure of the resulting GNRs because the orientation of graphene is unknown. More importantly, as considerable edge disorder exists owing to the harsh etching process, the carrier mobility of GNRs is greatly reduced. Considering the disordered edge structures formed by above-mentioned approaches, the carrier transport mechanism in these GNRs can be broadly explained by a thermally activated carrier hopping mechanism. Thus, top-down fabricated GNRs can be considered as a series of small graphene fragments with heavily disordered edge structures [29]. To obtain highquality GNRs with controlled edge structures, various approaches for fabricating GNRs without using harsh oxygen-plasma-assisted etching have been demonstrated.

\subsubsection{Hydrogen-assisted etching without masks}

Hydrogen is another option for graphene etching because carbon atoms can be removed from graphene edges by reaction with hydrogen gas catalyzed by metal nanoparticles, such as Ni, Fe, and Co [30-32], at elevated temperatures. Ajayan's group eported that graphene cutting can be promoted using a multistage cutting technique by lithographically prepatterning edge steps in graphene, resulting in improved edges and shape control [33]. Sub$10 \mathrm{~nm}$ GNRs and nanostructures, such as equilateral triangles, have been obtained using this catalytic etching method, which may open new avenues for studying novel electronic behavior owing to the crystallographically oriented edge. Catalytic etching with metal particles shows great potential for fabricating GNRs with crystallographically oriented edges. However, GNRs obtained using this method are often in disarray. Moreover, metal particle residues degrade the electrical per- 
(a) $\mathrm{Si} / \mathrm{HfO}_{2}$ coreshell nanowire on graphene

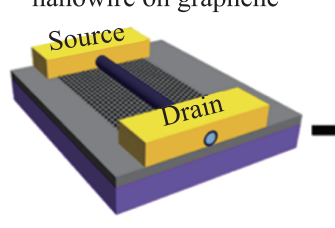

(b) $\mathrm{O}_{2}$ plasma etch to define GNR

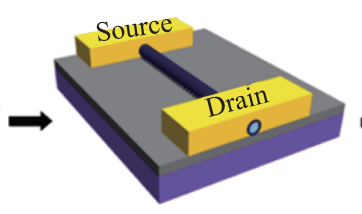

(c) Ar plasma to remove top half $\mathrm{HfO}_{2}$ shell

(d) Deposition of metal

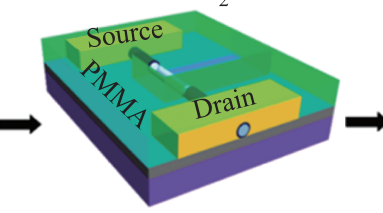

gate

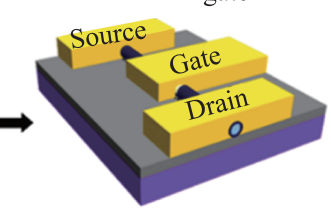

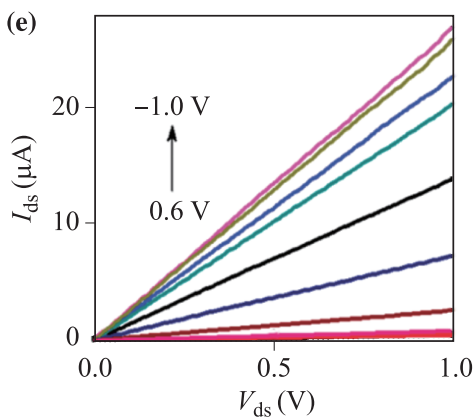
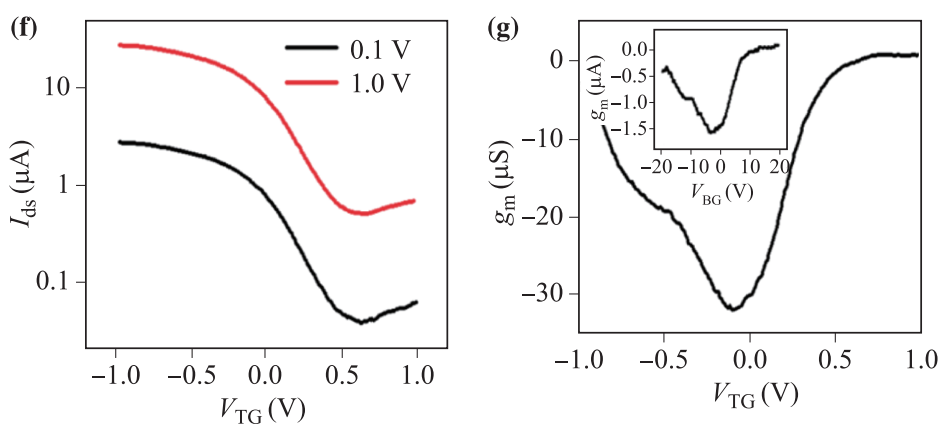

Fig. $3(\mathbf{a}-\mathbf{d})$ Procedure in top gate GNRs fabrication using $\mathrm{Si} / \mathrm{HfO}_{2}$ core/shell. (e-g) Electrical properties of the topgated GNR device. Reproduced from Ref. [19].

formance of GNRs, impeding their utilization in further electronic and transport research.

Hydrogen etching can also occur without the assistance of catalytic nanoparticles. Recently, our group has achieved anisotropic etching of the graphene basal plane via a facile hydrogen plasma etching technique [34, 35]. Our results show that zigzag edges are the most stable edge structures under hydrogen plasma etching, as shown in Fig. 4. The etching mechanism is attributed to hydrogenation and volatilization of carbon atoms, and the etching rate can be controlled by tuning the plasma intensity, temperature, and etching time. Notably, anisotropic hydrogen etching only occurs at edges and inherent defects and does not induce additional defects owing to the mild nature of hydrogen plasma, whereas the harsh nature of oxygen plasma usually introduces additional defects. GNRs with atomically smooth zigzag edges could be achieved by taking advantage of this anisotropic etching effect [35].

This fabrication approach consists of two main processes: first, creation of lithography-defined artificial defects; and second, enlargement of these artificial defects by anisotropic etching. Lithography-defined circular holes can act as artificial defects to seed hexagonal growth during anisotropic etching. This technique is favorable for obtaining scale-up patterned graphene nanostructures with controllable sizes and, more importantly, well-defined zigzag edges. In addition, various patterned graphene nanostructures can be obtained by utilizing this approach, such as honeycomb-like networks, arrays of isolated triangular dots, and waved ribbon arrays (Fig. 5).

GNRs with widths down to $6 \mathrm{~nm}$ can be obtained us- (a)

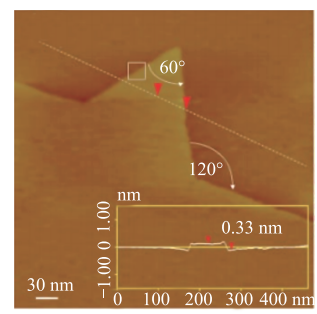

(c)

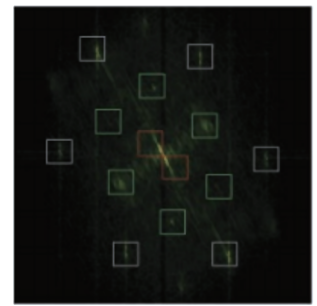

(b)

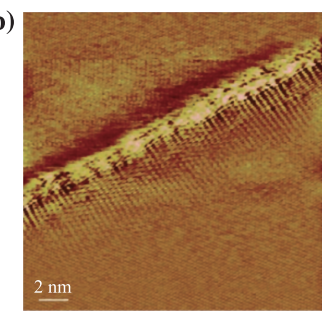

(d)

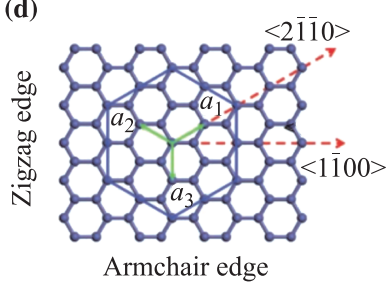

Fig. 4 Etching anisotropy in the graphite basal plane. (a) Typical constant current STM image of monolayer hexagonal pit on graphite. (b) Atomic resolution for the region marked in (a). (c) The Fourier transform spectrum. (d) Illustration of etching speed. Reproduced from Ref. [34].

ing this method. The on/off ratio is $\sim 3$ through $\pm 40 \mathrm{~V}$ at room temperature and is independent of width, indicating the quasi-metallic conduction behavior of our ZGNRs. The high quality of these GNRs is indicated by the high carrier mobility achieved of $\sim 2000 \mathrm{~cm}^{2} \cdot \mathrm{V}^{-1} \cdot \mathrm{s}^{-1}$ in GNRs with a width of $10 \mathrm{~nm}$, which is the highest reported value for GNRs with similar widths. For graphene on $\mathrm{SiO}_{2}$ substrates, this method is only suitable for bilayer and multilayer graphene owing to the surface roughness and charge inhomogeneity of $\mathrm{SiO}_{2}$ substrates. 
(a)

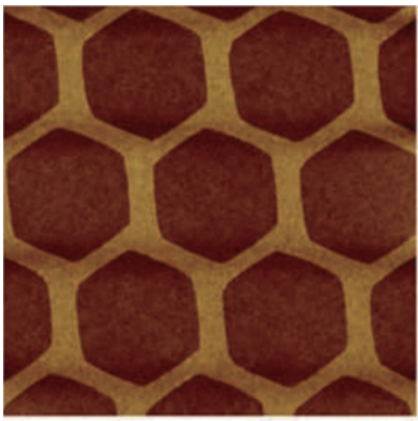

(c)

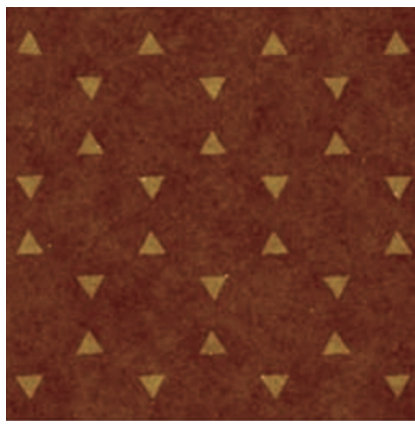

(b)

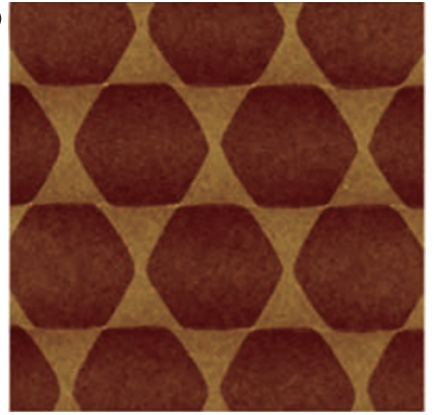

(d)

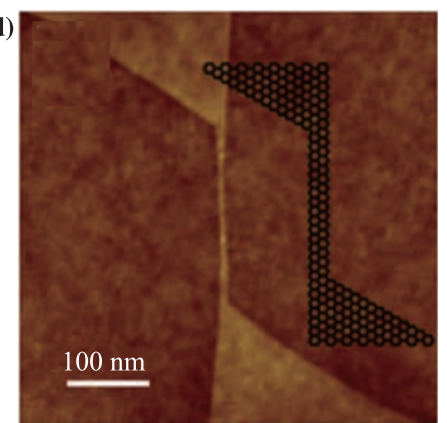

(e)

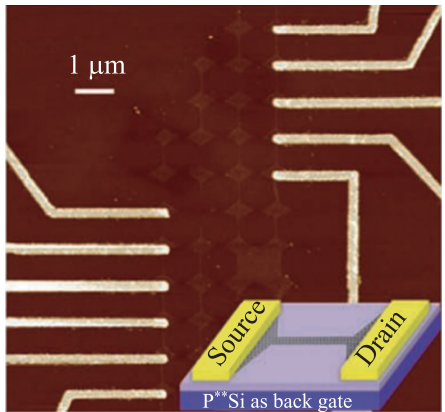

(f)

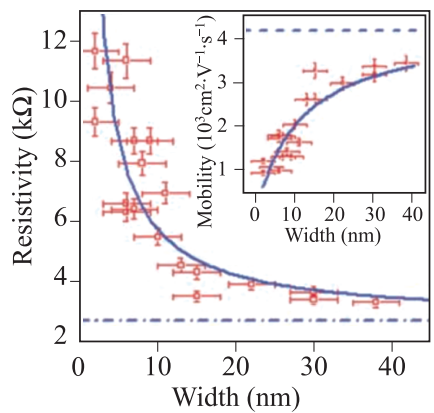

Fig. 5 Hydrogen-assisted anisotropic etching of various patterned graphene nanostructures. (a-c) Various patterned graphene nanostructures. (d) Zigzag-GNR with width $\sim 5 \mathrm{~nm}$. (e) AFM image of $10 \mathrm{~nm}$ ZGNRs devices. (f) Carrier mobility against ribbon width. Reproduced from Ref. [35].

Most recently, our group has optimized this etching process for monolayer graphene by using an atomically flat and inert hexagonal boron nitride (h-BN) substrate [36]. Mobilities up to $3000 \mathrm{~cm}^{2} \cdot \mathrm{V}^{-1} \cdot \mathrm{s}^{-1}$ can be achieved for ZGNRs with a width of $25 \mathrm{~nm}$, demonstrating their high quality (Fig. 6). This method provides a simple and controllable way to fabricate large-scale GNRs with welldefined, uniform widths and atomically smooth zigzag edges.

\subsubsection{Scanning probe lithography}

Scanning probe lithography (SPL) is another straightforward approach for patterning graphene with controlled crystallographic orientation and fine structure down to several nanometers. As a kind of SPL, atomic force microscopy (AFM)-based local anodic oxidation (LCO) lithography has been used to fabricate GNRs [37-40]. AFM-based LCO lithography has the capability of fabricating small features. However, the downscale features are limited by the radius of the tip and the edge chirality cannot be tailored specifically.

To avoid the limitations of AFM-based LCO lithography, Tapasztó et al. used scanning tunneling microscopy (STM) lithography to pattern graphene by applying a bias potential and simultaneously moving the STM tip to etch the unwanted area [41]. It is possible to cut GNRs with specific edge chirality by adjusting the lithographic parameters, such as the bias potential and tip velocity (Fig. 7). Beyond the precise engineering of the edge structure in STM lithography, another advantage is the possibility for downscaling. Strikingly, GNRs with widths down to $\sim 2.5 \mathrm{~nm}$ and energy gaps up to $0.5 \mathrm{eV}$ can be obtained. Further, the local density of states (LDOS) of the predefined GNRs can be detected in situ to unveil physical phenomena.

Beam-induced cutting and reshaping of graphene, called beam lithography, is another way to pattern graphene down to few nanometers. Beam lithography employs the focused ion beam in transmission electron microscopy (TEM) [42] or helium and neon ions in scanning ion beam microscopy $[43,44]$. Both methods are suitable for producing graphene patterns in the range of tens of nanometers, but damage introduced by beam lithography cannot be avoided and the edges of the patterned samples are quite rough. However, these approaches can provide a facile way of cutting graphene into desired patterns with high efficiency.

\subsubsection{Unzipping of carbon nanotubes}

Another promising approach to obtain GNRs for mass production is the unzipping of carbon nanotubes $(\mathrm{CNTs})$ $[8,9]$. In this top-down method, CNTs act as the starting material, and the width of the produced GNRs is determined by the circumference of the CNTs. Tour et al. [9] obtained GNRs by the lengthwise unzipping of multiwalled CNTs through treatment with sulfuric acid 
(a)

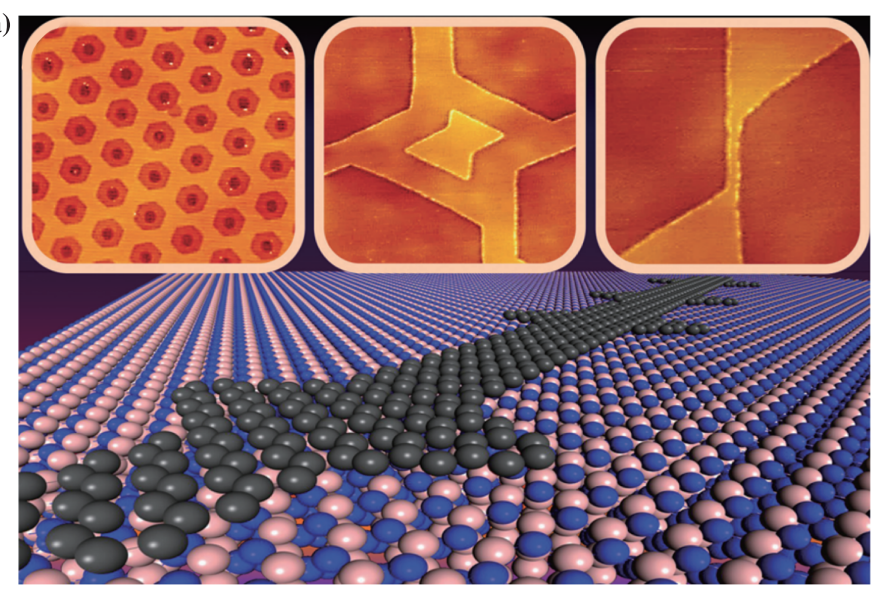

(b)
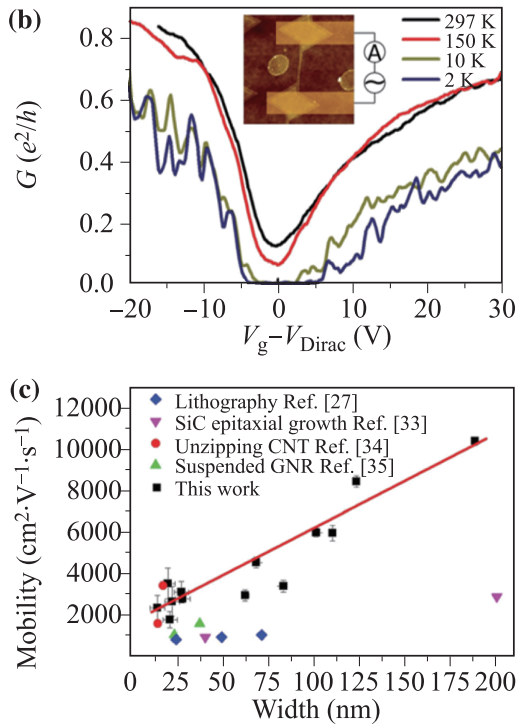

Fig. 6 Patterning of monolayer graphene on h-BN. (a) AFM images of patterned monolayer graphene nanostructures. (b) Measured conductance of ZGNR at various temperature. (c) High mobility of the patterned ZGNRs. Reproduced from Ref. [36].

(a)

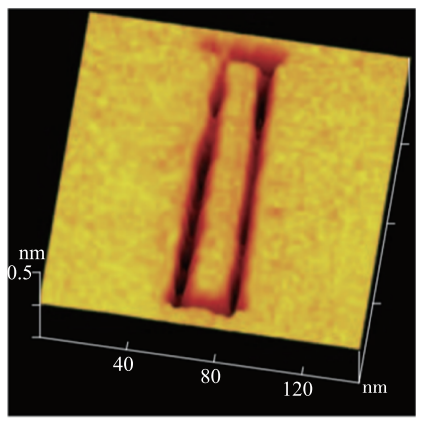

(b)

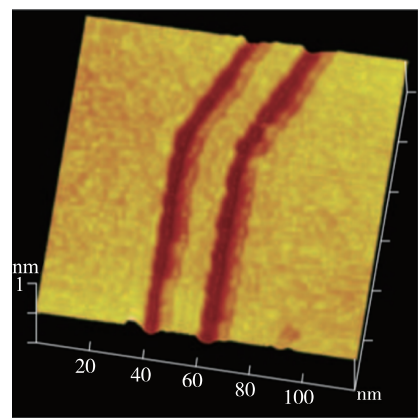

(c)

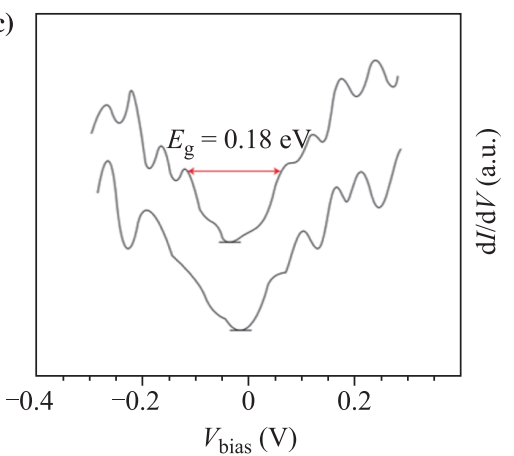

Fig. 7 GNR patterned by STM lithography. (a) 3D STM image of GNRs with $10 \mathrm{~nm}$ width and $120 \mathrm{~nm}$ length. (b) A 30 GNR junction with $8 \mathrm{~nm}$ width connecting armchair GNRs and zigzag GNRs. (c) STS of the obtained ribbon. Reproduced from Ref. [41].

and potassium permanganate. However, the quality of these GNRs was low owing to the presence of oxygencontaining functionalities at the edge and on the surface [45]. Various improved methods have been described for enhancing the quality of GNRs by reducing the oxidative edge defects caused by chemical unzipping.

Unlike the oxidative method mentioned above, Dai's group developed the oxidative method mentioned for obtaining GNRs with a narrow width distribution (10-20 $\mathrm{nm}$ ) and smooth edges [8]. CNTs were first embedded in poly (methyl methacrylate) (PMMA), which acted as an etch mask, and then argon plasma was used to remove a longitudinal strip of carbon atoms from the side walls of the CNTs, as shown in Fig. 8. The edges of the obtained GNRs were smooth at the resolution of AFM imaging. The width distribution of the obtained GNRs was 10$20 \mathrm{~nm}$, and narrower GNRs could be obtained utilizing this method by using CNTs with narrower diameters. In addition, Dai's group constructed well-ordered arrays of GNRs and GNR-CNT crossbars by unzipping CNTs [46]. Recent progress in the synthesis, size control, and alignment control of CNTs in combination with argon plasma unzipping of CNTs might pave the way to fabricating GNR arrays in a controlled manner. As argonassisted unzipping of CNTs is limited to the formation of GNRs on the substrate, the same research group developed a two-step method to obtain high-quality GNRs [46]. The quality of the achieved GNRs was exemplified using aberration-corrected TEM and quantum transport measurements, including Coulomb blockade, Kondo effect, and inelastic cotunneling $[47,48]$. Because of their high quality, these samples show potential for exploring new physics, such as magnetic edge states [49] and spin qubits [50]. 
(a)
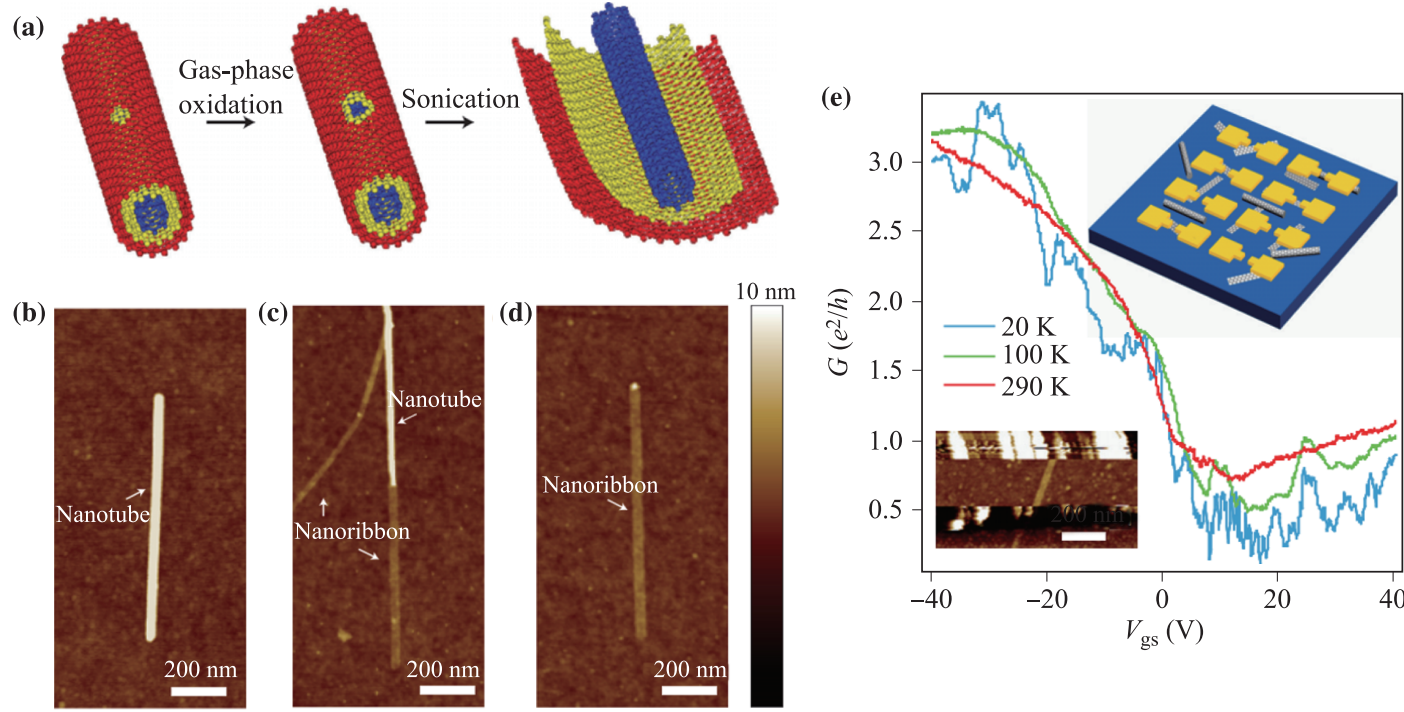

Fig. 8 GNRs obtained by lengthwise unzipping of CNTs. (a) Schematically illustration of GNRs formation. (b-d) TEM images demonstrating the transformation from multiwall CNTs (left) to oxidized GNRs (right). (e) Electrical transport measurement of the obtained GNR. Reproduced from Ref. [8].

While these unzipped GNRs show promise, their structures depend highly on the raw CNT materials, and obtaining of GNRs with well-defined edge structures is very difficult to achieve as CNTs have many different chiralities.

\subsection{Bottom-up approaches}

In addition to the various top-town approaches mentioned above for fabricating GNRs, another route for GNR fabrication is the bottom-up approach, which is briefly summarized in the following sections.

\subsubsection{Self-assembly of organic molecules}

Self-assembly of organic molecules using solutionmediated $[51,52]$ or surface-assisted cyclodehydrogenation reactions [53] is a promising method for obtaining GNRs with ultra-narrow widths. This method was first reported by Cai et al. [53], who used surface-assisted coupling of molecular precursors into linear polymers to obtain AGNRs (Fig. 9). Recently, the same group reported the fabrication of ZGNRs by choosing a different precursor [54]. This method requires two thermal activation steps, described as dehalogenation and cyclodehydrogenation, which are achieved by annealing the samples at different temperatures. Notably, different types of GNRs can be obtained by choosing different molecular precursors. This method may even provide a route to the controlled growth of GNR heterojunctions by using two different monomers designed for facile heteromolecule coupling and cyclodehydrogenation. The same group fabricated intraribbon heterojunctions and
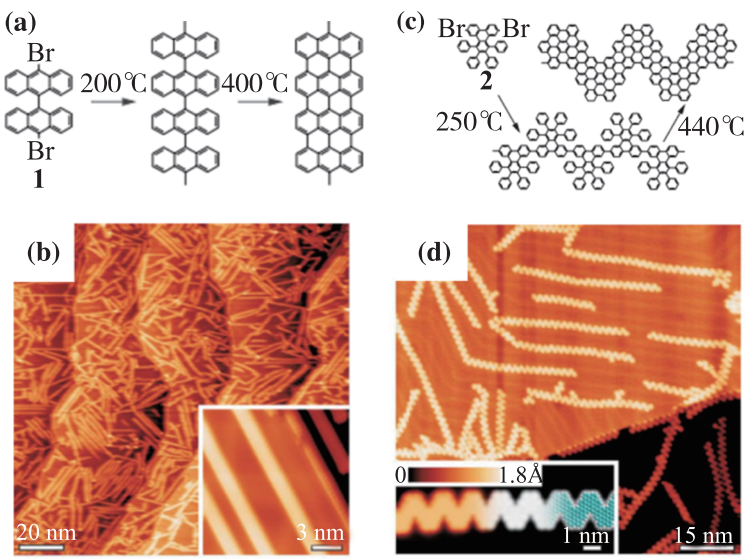

Fig. 9 GNRs formation through surface-assisted. (a) Reaction scheme to form $N=7$ straight GNRs. (b) STM images of the GNRs on $\mathrm{Au}(111)$ surface. (c) Reaction scheme to form chevron-type GNRs. (d) STM images of the GNRs on $\mathrm{Au}(111)$ surface. Reproduced from Ref. [53].

PN graphene heterojunctions via partial cyclodehydrogenation of monomers, which was controlled using different annealing temperatures or by combining the pristine precursors with their nitrogen-substituted equivalents $[55,56]$. Later, Crommie and co-workers fabricated 7 13 GNR heterojunctions using the surface-assisted bottom up approach by choosing different molecular blocks [57]. STM and scanning tunneling spectroscopy (STS), as well as first-principle calculations, revealed molecularscale band engineering $[58,59]$. These GNRs have atomically well-defined structures and large band gaps are detected owing to quantum confinement. 
Besides the above-mentioned precursors, a number of studies have been based on the solution synthesis of GNRs from polyphenylene [51, 52, 60]. Most recently, Müllen and co-workers developed a new bottom-up solution synthesis method via Diels-Alder polymerization [52]. GNRs with lengths of more than $200 \mathrm{~nm}$, the longest reported to date for self-assembly of organic molecules, can be synthesized by employing this approach. However, these GNRs require post-synthesis transfer to other substrates through the above-mentioned surface-assisted or solution-mediated methods in order to be used for device fabrication. Moreover, these ribbons are too small to investigate their transport properties.

\subsubsection{Growth of graphene nanoribbons on templated substrates}

A major limitation of the bottom-up synthesis of GNRs by self-assembly of organic molecules lies in the difficulties in fabricating GNRs on insulating substrates. Growth of GNRs directly on insulating substrates is more favorable, as further investigation of their electronic properties is straightforward. Epitaxial growth of graphene on silicon carbide and chemical vapor deposition (CVD) growth of graphene on catalyst substrates are two of the most popular methods for waferscale graphene production [5,61]. A common feature of these methods is that the substrate guides the growth of graphene, as the morphology of graphene is highly in- fluenced by the structure of the underlying substrate. Therefore, bottom-up growth of GNRs on templated substrates is extremely promising. The exploitation of direct growth of GNRs on templated silicon carbide substrates was first demonstrated by de Heer's group (Fig. 10) [62]. GNRs as narrow as $40 \mathrm{~nm}$ can be grown at specified positions on the substrate. The as-grown GNRs exhibit on/off ratios of $\sim 10$ and mobilities of up to $2700 \mathrm{~cm}^{2} \cdot \mathrm{V}^{-1} \cdot \mathrm{s}^{-1}$ at room temperature. Importantly, pretailoring of silicon carbide substrates, in general, is a promising approach for fabricating high densities of GNR devices. Soon after, single-channel ballistic transport at room temperature was observed in epitaxial GNRs on silicon carbide, a signature of the high quality of such GNRs [4].

Cho and co-workers presented another facile and effective approach for fabricating width-controlled GNRs in a more efficient way [63]. By tuning the annealing temperature and gas pressure, steps can be formed on the surface of silicon carbide and used for GNR growth. Recently, Nevius et al. showed asymmetric growth of armchair- and zigzag-terminated GNRs from the different silicon carbide step facets [64]. Further, the desired chirality of preferential precipitated GNRs can be obtained by selecting an appropriate silicon carbide sidewall facet.

CVD is a powerful and effective approach for growing monolayer graphene on catalytic metal substrates. Through patterning of the catalytic metal substrates, (a)

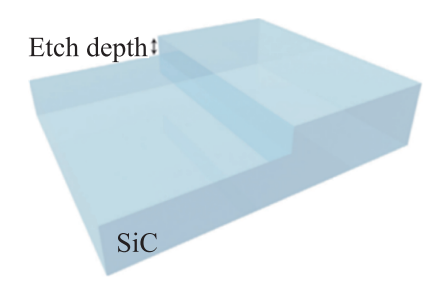

(c)

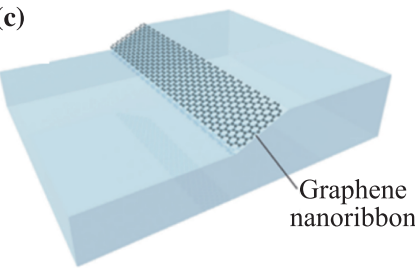

(b)

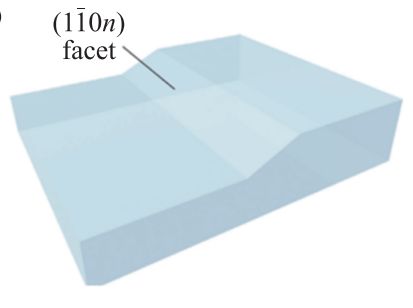

(d)

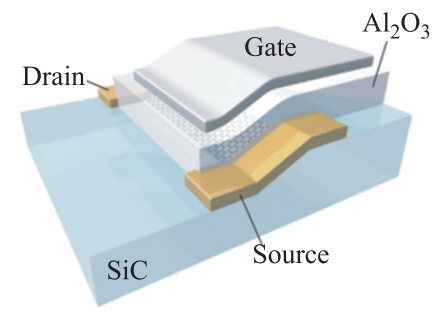

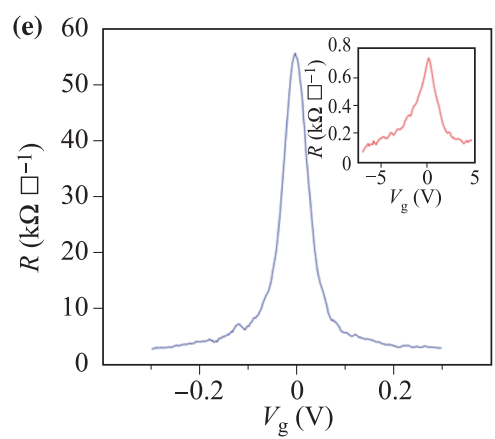

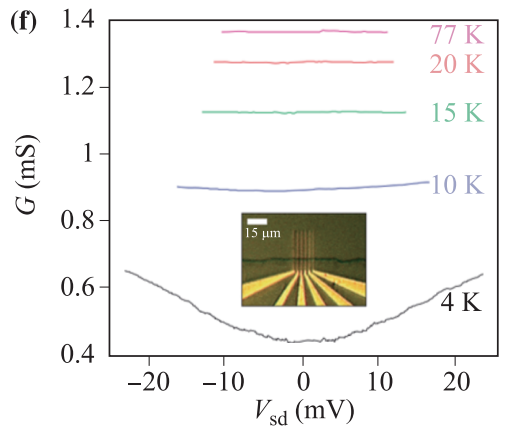

Fig. 10 Scalable templated growth GNRs on SiC. (a, b) Etch to form SiC step and specific facet. (c) Self-organized GNRs formation in the step facet. (d) Illustration of top gate GNRs device. (e, f) Electrical properties of the obtained GNR. Reproduced from Ref. [62]. 
it is also possible to directly grow GNRs. Metal catalysts that having steps or twin boundaries are reported to stimulate GNR growth [65]. In addition to Co and $\mathrm{Cu}, \mathrm{Ni}$ can also act as a catalyst for GNR growth. To date, several routes based on $\mathrm{Ni}$ catalysts for direct growth of GNRs have been reported [66-68]. In their pioneering work, Kato and Hatakeyama achieved site- and alignment-controlled growth of GNRs by directly converting Ni nanobars into GNRs via rapid heating plasma CVD (RH-PCVD) [66]. The widths of the obtained GNRs depend on the initial width of the Ni nanobar, and more importantly, do not exceed the width of the $\mathrm{Ni}$ nanobar, indicating the formation of width-controlled GNRs, as shown in Fig. 11.

In addition to Ni nanobars, vertical and planar Ni films can act as catalyst films to template GNR growth. Zhang and co-workers demonstrated precise control of the position and width of GNRs based on surface catalytic selectivity at the vertically $\mathrm{Ni}$ template, with the width defined by the thickness of the catalyst film [67]. The obtained GNRs have widths as narrow as $20 \mathrm{~nm}$ and show high carrier mobilities of up to $1000 \mathrm{~cm}^{2} \cdot \mathrm{V}^{-1} \cdot \mathrm{s}^{-1}$. Recently, Hibino and co-workers reported the catalytic growth of GNRs on a planar Ni film [68]. GNRs with widths of 20-30 nm can be formed by annealing a thin $\mathrm{Ni}$ film, deposited with PMMA, at $1000{ }^{\circ} \mathrm{C}$.

Jacobberger et al. reported the direct growth of AGNRs on the insulating substrate germanium. This method allows fabrication of GNRs down to $10 \mathrm{~nm}$ in width with smooth armchair edges and controlled crystallographic orientation [69].

Recently, our group exploited the epitaxy of GNRs on h-BN substrates to obtain GNRs with widths ranging from 15 to $150 \mathrm{~nm}$ [70]. Strikingly, the as-grown GNRs on h-BN show ultra high quality with a carrier mobilities of up to $\sim 20000 \mathrm{~cm}^{2} \cdot \mathrm{V}^{-1} \cdot \mathrm{s}^{-1}$ at low temperature and $\sim 4000 \mathrm{~cm}^{2} \cdot \mathrm{V}^{-1} \cdot \mathrm{s}^{-1}$ at room temperature owing to effective reduction of charge impurities and phonon scattering for epitaxy on the atomically smooth h-BN surface (Fig. 12). Moreover, this growth approach provides an ideal platform to investigate GNRs with clean interfaces as well as quasi-one-dimensional superlattice systems.

\subsection{Challenges in electronic-grade graphene nanoribbon processing}

Sections 2.1 and 2.2 summarized the reported methods for GNR fabrication. In addition to different scalability and controllability, each of these methods generates graphene nanostructures with different widths and edge structures, which greatly influence their electronic properties, such as energy band gap, on/off ratio, and carrier mobility.

Table 1 lists the feature size, on/off ratio, band gap, and edge structure of the various types of GNRs. The feature size is characterized by the narrowest width of GNRs fabricated by a certain method. To utilize GNRs
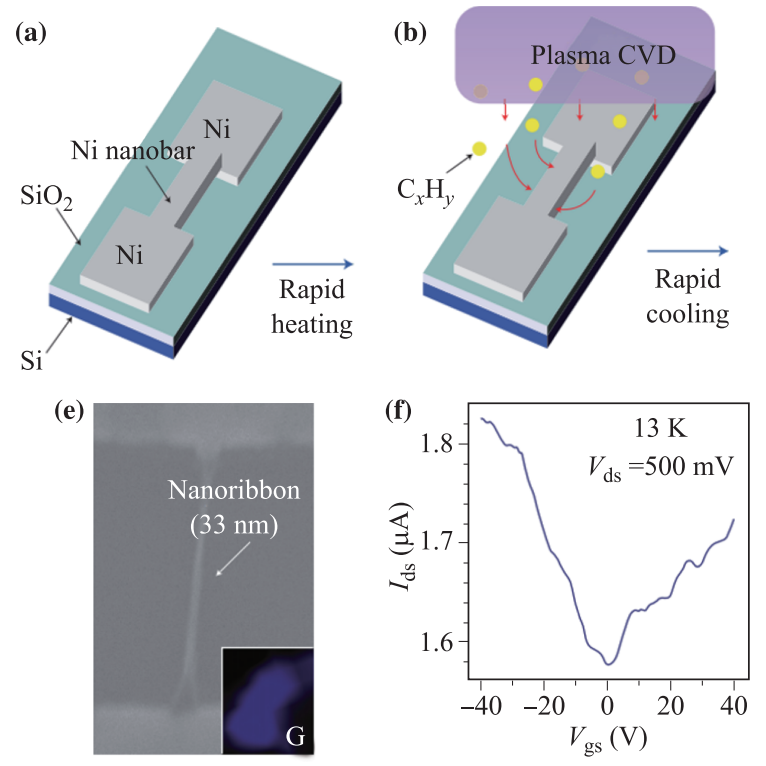

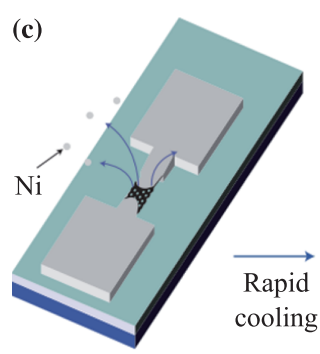

(d)

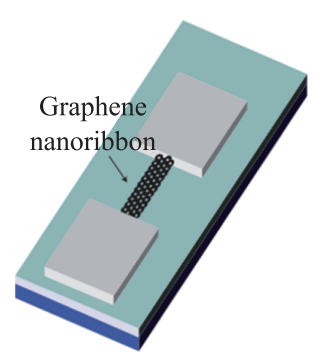

(g)

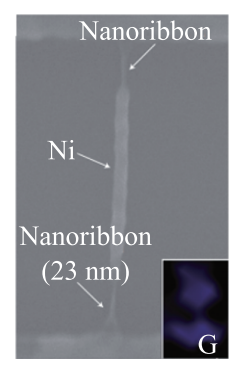

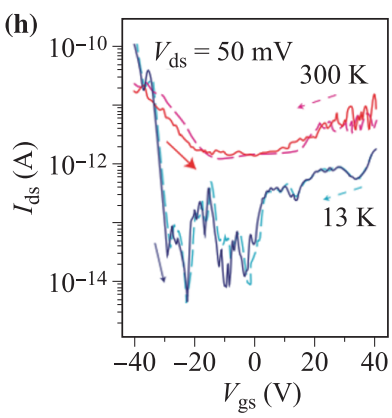

Fig. 11 Direct conversion of Ni nanobar to GNRs via PH-PCVD. (a-d) Schematic illustration of GNRs formation. (e, f) SEM image of $33 \mathrm{~nm}$ GNR and its electrical performance. (g, h) SEM image of $23 \mathrm{~nm}$ GNR and its electrical performance. Reproduced from Ref. [66]. 

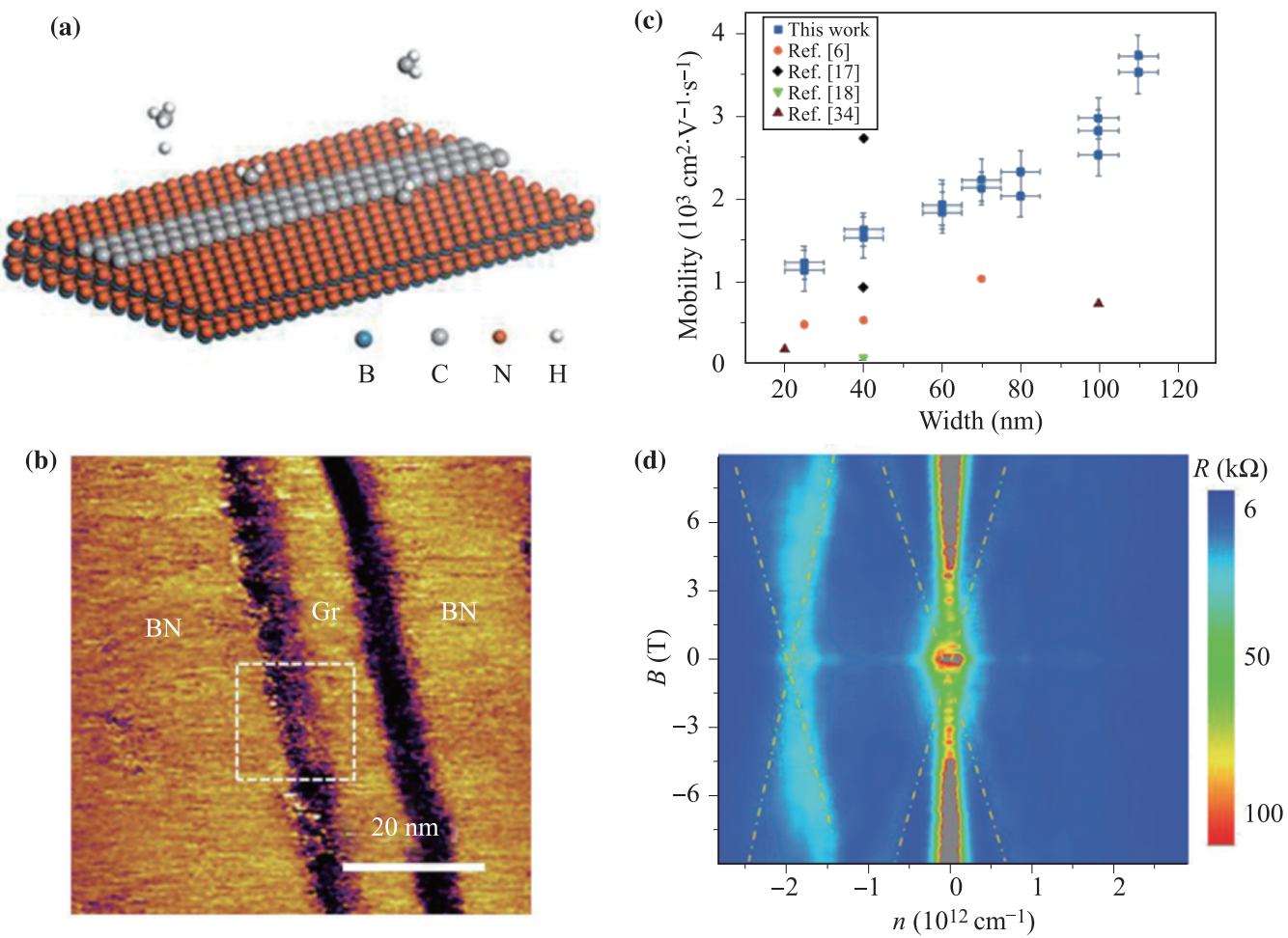

Fig. 12 Epitaxy of GNRs on h-BN substrate. (a) Schematic illustration of growth mechanism. (b) Contact mode AFM image of $15 \mathrm{~nm}$ GNRs. (c) Statistic mobility of as growth GNRs versus ribbon width. (d) Two-terminal magnetoresistance. Reproduced from Ref. [70].

as semiconductor devices at room temperature, a technologically relevant band gap that is substantially greater than $k_{B} T=26 \mathrm{meV}$ is required, while preserving high carrier mobility. Numerous efforts have been dedicated to induce band gap in GNRs using quantum confinement. The band gaps of GNRs caused by quantum confinement are inversely proportional to the width of the ribbons, as shown in Table 1. Sizable band gaps as large as $500 \mathrm{meV}$ can be achieved.

To utilize GNRs in logic devices, a width-controlled band gap is not sufficient, as the carrier mobility, which is related to the smoothness of the edge structures, also needs to be considered. Figure 13 shows the carrier mobilities of GNRs obtained using the reported approaches. The carrier mobilities of GNRs are smaller than that of pristine graphene flake owing to edge scattering. Rougher edge structures decrease the carrier mobility and thus reduce the quality of GNRs. Considering these two characteristics, the current methods have not shown great potential for utilizing GNRs as logic devices at room temperatures. However, GNRs with zigzag edge termination show great potential for application in spintronics as well as valleytronics, as already proposed by theorists and experimental researchers. High-quality GNRs with well-defined edges are the ideal platform for further explorations of electronic and transport properties. Therefore, fabrication of GNRs with defined edge terminations and controllable widths that maintain high carrier mobility is still a challenging and interesting research topic.

\section{Electronic band structure of graphene nanoribbons}

3.1 Differences between the band structures of zigzag and armchair nanoribbons

Based on the edge structures, GNRs have two typical edge terminations: armchair and zigzag, as shown in Fig. 1. Theoretically, the electronic properties depend strongly on the size and geometry of the GNRs [71, 72]. The electronic states may be understood in terms of eigenstates of the Dirac Hamiltonian with appropriate boundary conditions [73]. The energy spectra and wavefunction for zigzag and armchair edges have been widely explored in theoretical studies. Herein, we briefly review the electronic states of ZGNRs and AGNRs.

For AGNRs, the ribbon width determines whether the ribbon is metallic or semiconducting. When the ribbon 
Table 1 Feature size, on/off ratio and band gap of GNRs produced by different methods.

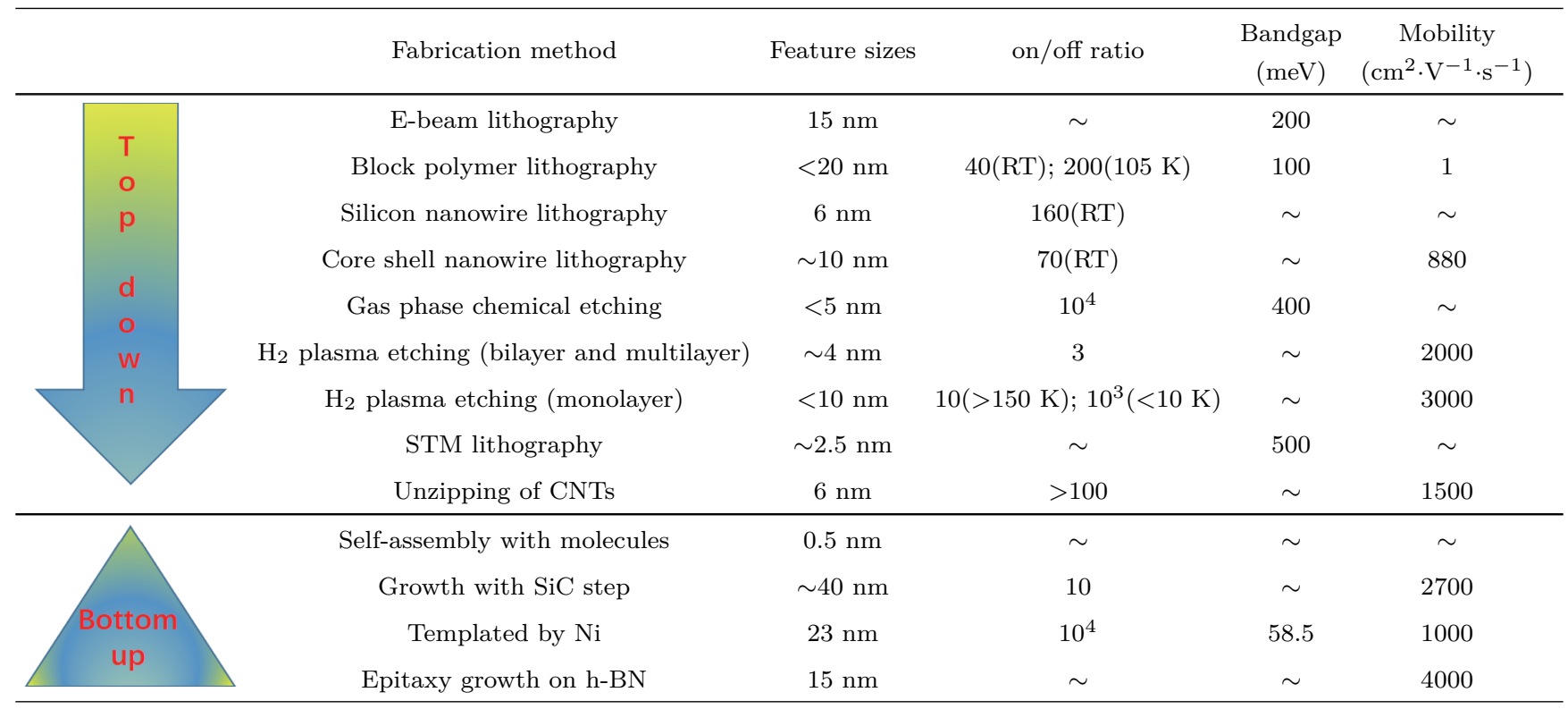

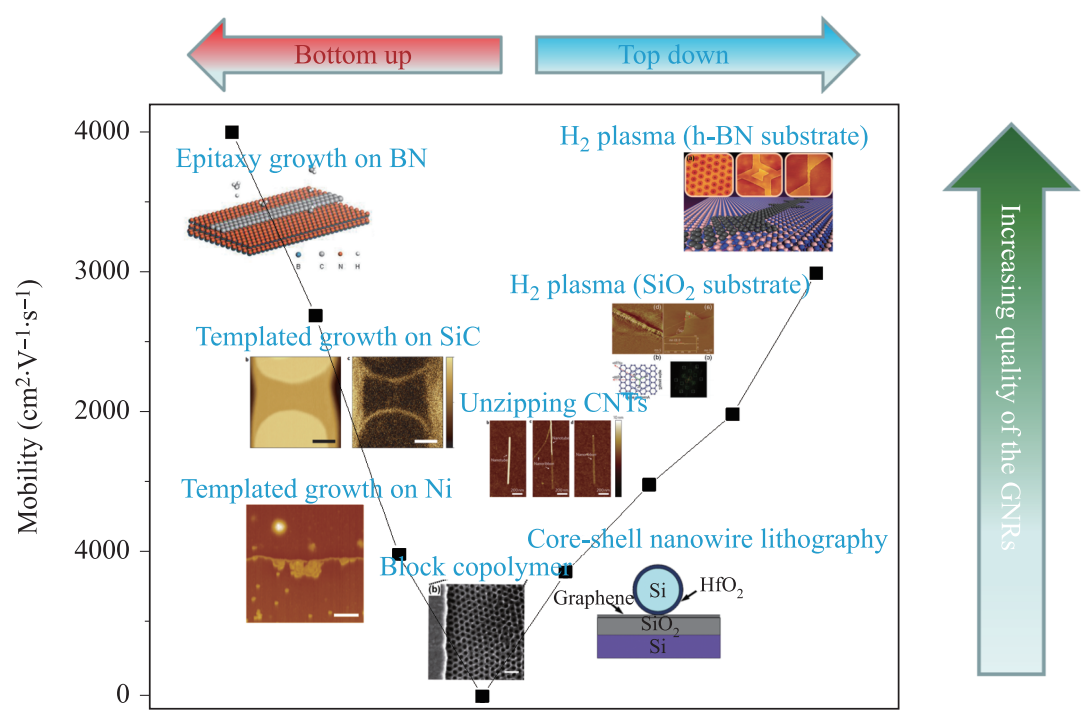

Fig. 13 Comparison of the mobility of the GNRs from the reported approaches.

width $N=3 M-1$, where $M$ is an integer, the system is metallic. Otherwise, the ribbons show insulating characteristics. For semiconducting nanoribbons, the direct band gap decreases as the ribbon width increases, reaching zero at the limit of very large $N$.

However, for ZGNRs, a remarkable feature arises in the band structure, as the highest valence band and lowest conductance band are always degenerate at $k=\pi$, which does not originate from the intrinsic band structure of graphene. The electronic states in the almost flat band can be understood as a localized state on the zigzag edge, as indicated by the charge density distribution [71].

\subsubsection{Edge state in zigzag graphene nanoribbons}

Theoretically, ZGNRs exhibit a special edge state, which corresponds to partly flat bands [71]. These partly flat bands were first analyzed by Fujita et al. by using tightbinding band calculations [72]. A perfectly flat band can be constructed within the k-space region $\frac{2 \pi}{3} \leq|k| \leq \pi$. The edge state were further demonstrated to be a robust property of zigzag edges and non-negligible edge states 
can survive in ZGNRs, even with less-developed zigzag edges $[71,74]$. Calculations showed that edge states are exhibited when then average number of sequential zigzag sites reaches four or five.

The energy dispersion of ZGNRs can be calculated by the following formula [75]:

$$
E_{k}=-2 t N D_{k}^{N-1}\left(-2 t+2 t \cos \frac{k}{2}\right),
$$

where $D_{k}=-2 \cos (k / 2)$ and $\mathrm{N}$ represents the ribbon width. The density of states (DOS) can be calculated from the dispersion relationship,

$$
\rho(\varepsilon)=\frac{\partial k}{\partial \varepsilon} \sim \frac{1}{N} \varepsilon^{\alpha},
$$

where $\alpha=1 / N-1$. Notably, the DOS gives a remarkably sharp peak related to the edge state at the Dirac point. The DOS of ZGNRs with different widths $(N=6$, 11 , and 50, corresponding to widths of $1.1,2.2$, and 10.6 $\mathrm{nm}$, respectively) are shown in Fig. 14.

Zigzag edges are formed by atoms that belong to the same sublattice: A or B. The partly flat band is of great significance for the properties of ZGNRs. Theoretical studies have shown that a flat band can be constructed by an imbalanced number of sublattice $\mathrm{A}$ and $\mathrm{B}$ atoms, that is $\left|N_{A}-N_{B}\right| \neq 0$, where $N_{A}$ and $N_{B}$ represent the number of A and B sublattice sites, respectively [76]. Recently, it was shown that zero-energy states generally appear when the potential between the A and B sublattices is strongly imbalanced, which can be generated from edges or defects, even at $\left|N_{A}-N_{B}\right|=0$. Wakabayashi et al. found that the partly flat band still occurs at zero energy even under the condition of $\left|N_{A}-N_{B}\right|=0$ when edge structures are partially modified [77]. Moreover, the modification of AGNRs also leads to a zero energy state and partly flat band. These results pave the way for designing and stabilizing the edge state by chemical and structural modification of graphene edges.

Fujita et al. analyzed the magnetic structure of GNRs using the Hubbard model with an unrestricted HartreeFock approximation [71]. A local ferromagnetic structure

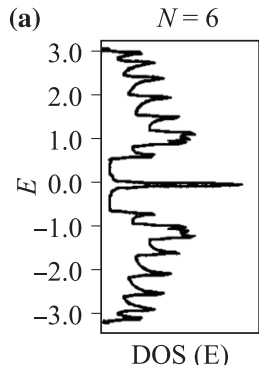

$\operatorname{DOS}(\mathrm{E})$

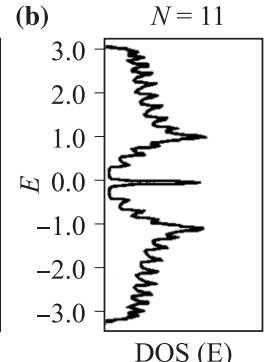

DOS (E)

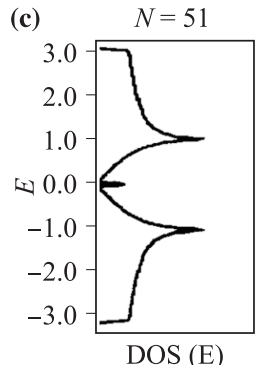

DOS (E)
Fig. 14 The DOS of zigzag GNR with different width. (ac) $N=6,11,51$. Reproduced from Ref. [75]. is found to exist at the zigzag edge and spin alignment is visualized at both edge sites, but AGNRs do not show similar magnetic behavior. The ground state of a ZGNR is antiferromagnetic, that is the spin-up and spin-down states belong to atoms in different edge sublattices. The unique magnetic properties of ZGNRs ensure that they are the most promising candidates for spintronics [49].

\subsubsection{Energy gap engineering in graphene nanoribbons}

Theoretical analysis showed that the band gap opening of GNRs depends on the edge orientation [72, 78]. Based on the tight-binding model within the nearest-neighbor or Hückle approximation, ZGNRs show metallic characteristics, regardless of their widths. Interestingly, the ribbon width is critical for determining whether an AGNR is metallic or semiconducting. These results show that there is a remarkably similarity between GNRs and CNTs [78]. ZGNRs and AGNRs are analogous with armchair CNTs and zigzag CNTs, respectively. Namely, all ZGNRs are metallic, as are all armchair CNTs, whereas AGNRs with a period of 3 are metallic, as are zigzag CNTs with a period of 3 . Barone et al. showed that the band gap of AGNRs greatly depends on the width [79]. To obtain GNRs with a band gap comparable to that of silicon, the width of the ribbons must be reduced to 1-2 $\mathrm{nm}$.

As the aforementioned models are based on simple tight-binding calculations, more detailed considerations of the edge effect are needed. Strikingly, Louie et al. employed first-principle calculations using the local density approximation (LDA) to analyze the band gap of GNRs [80]. Their results showed that both ZGNRs and AGNRs have energy gaps, with the value of the energy gap inversely proportional to the width of the nanoribbon (Fig. 15). The energy gaps of GNRs with different edge terminations are attributed to different physical mechanism. More specifically, the energy band gaps of AGNRs originate from quantum confinement while those of ZGNRs are caused by staggered sublattice potentials arising from magnetic ordering. Unlike the calculations based on the tight-binding approximation, the band gaps of AGNRs calculated using the LDA all correspond to semiconductor states, with the energy gap decreasing as the width of the AGNR increases, and no metallic states exist.

For ZGNRs, the results of the calculations using the LDA are shown in Figs. 16(a)-(c). Unlike the previous studies based on the tight-binding approximation or solutions of these calculations showed that ZGNRs have energy gaps analogous to those of AGNRs. The energy gaps of ZGNRs originate from staggered sublattice potentials resulting from magnetic ordering, as opposite spin states on opposite edges are located in different sublattices. The theoretical work of Louis's group demon- 

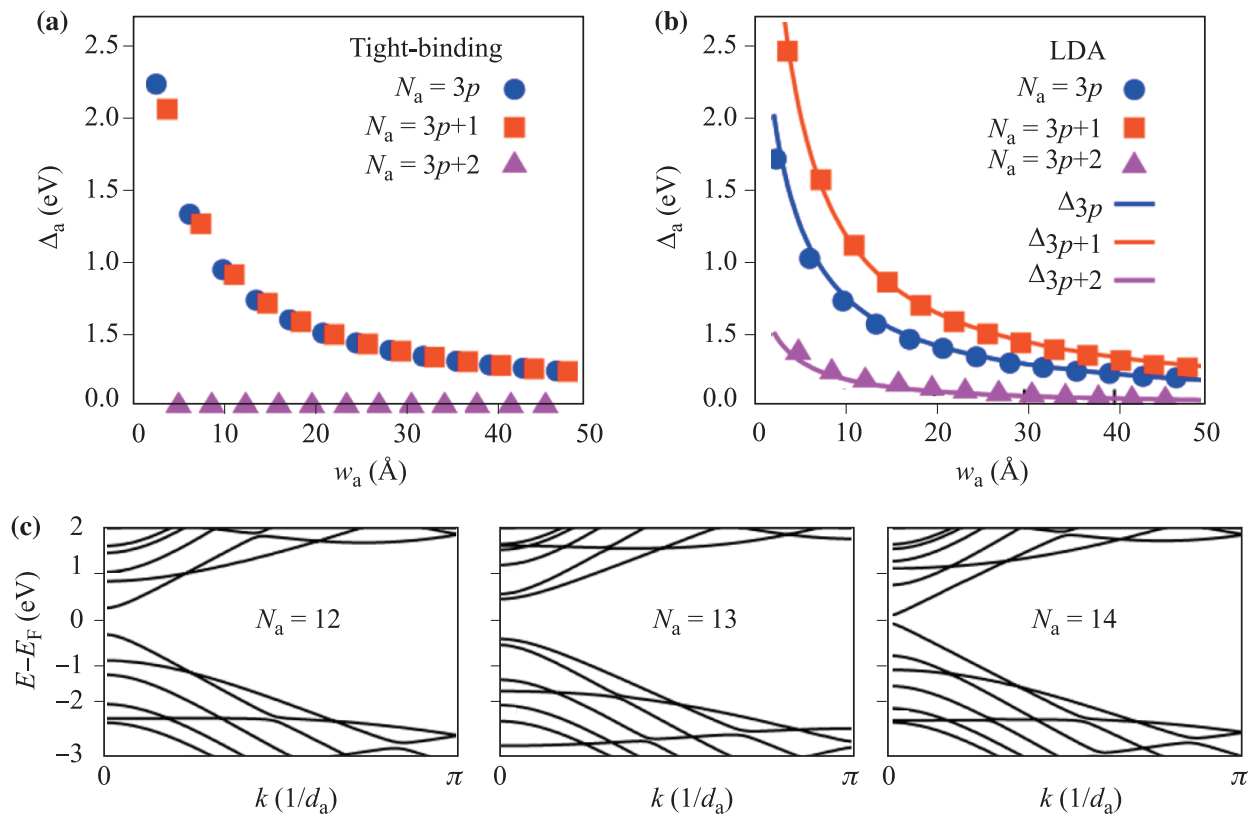

Fig. 15 (a, b) band gap of AGNRs in tight-binding model and LDA, respectively. (c) Band structure of AGNRs with $N_{\mathrm{a}}=12,13,14$, respectively in first principle calculation. Reproduced from Ref. [80].
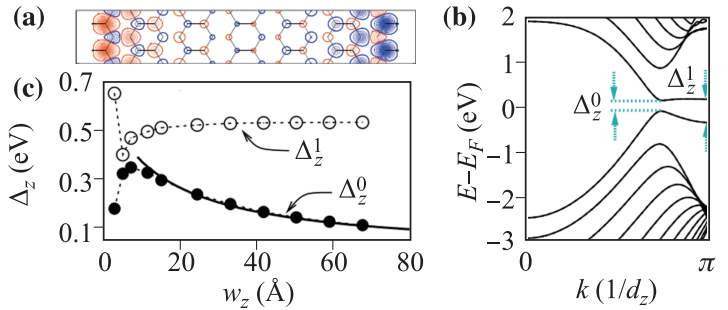

Fig. 16 (a) The spatial spin distributions in $N=12$ ZGNRs. (b) Band structure of $N=12$ ZGNRs. (c) The variation of $\Delta_{z}^{0}$ and $\Delta_{z}^{1}$ as a function of ribbon width. Reproduced from Ref. [80].

strates that the edge effect is a determining factor for band gap engineering in GNRs.

\subsection{Scanning tunneling microscopy and spectroscopy observations of edge states and band gaps}

\subsubsection{Zigzag edge states}

Localized zigzag edge states have been extensively studied, with a substantial amount of experimental work devoted to measuring graphene terrace edges using STM and STS [81-84]. STS measurements are performed to determine the electronic properties of graphene, as the $\mathrm{d} I / \mathrm{d} V$ quantity is proportional to the LDOS. The LDOS depends on atomic structure and it is a key to understanding the unconventional electronic structure of graphene. Therefore, direct observation of the local electronic structure near the edges of graphene using
STS measurements is of great significance for clarifying the edge states. In addition, the atomic resolution of graphene edges achieved in STM measurements can provide more detailed information about the edge state from the experimental perspective. Electronic DOS near the zigzag edges clearly show a LDOS peak that is several hundreds of meV above or below the Fermi level energy. Further, the complicated structures of the edges and charge transfer from different chemical functional groups bound to the edges of various samples might be responsible for discrepancies in peak energy $[81,83]$. STS data for zigzag and armchair edges are shown in Figs. 17(a)(d) [83]. A clear peak in the LDOS that is several tens of meV below the Fermi level energy is revealed for zigzag edges and its magnitude depends on the distances from the edge. The spatial decay of the edge state can be viewed as the peak magnitude decreasing, with the peak finally vanishing when scanning from the zigzag edge to the interior of graphene. Using STS measurements, Niimi et al. determined that the decay length of the edge state is about $1.2 \mathrm{~nm}$, in accordance with that calculated for a zigzag edge that is slightly mixed with an armchair edge [83]. In contrast, for the armchair edge, no obvious peak is observed in the LDOS data within experimental error.

Therefore, the LDOS peaks experimentally observed near the zigzag edge are indicative of the edge state in zigzag edge graphene, which has been intensively studied theoretically. Most recently, Ritter et al. investigated the role of edge states in a mixed edge employing ultra-high-vacuum STM and STS techniques [84]. The 
(a) Zigzag edge (ZYX)

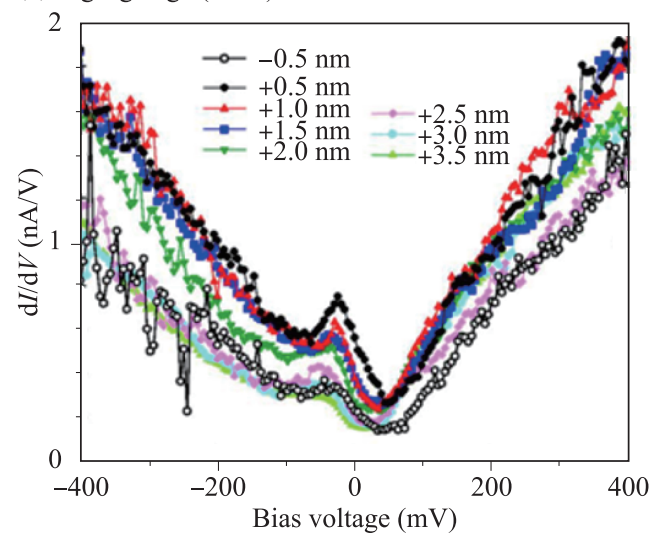

(c) Armchair edge (ZYX)

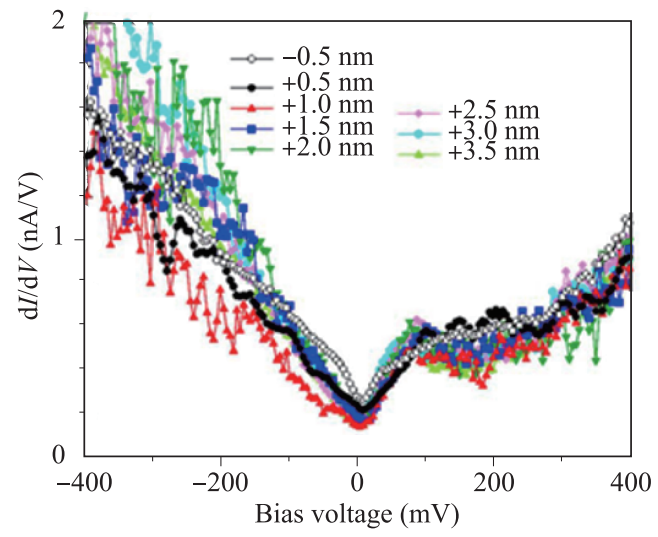

(b) Zigzag edge (HOPG)

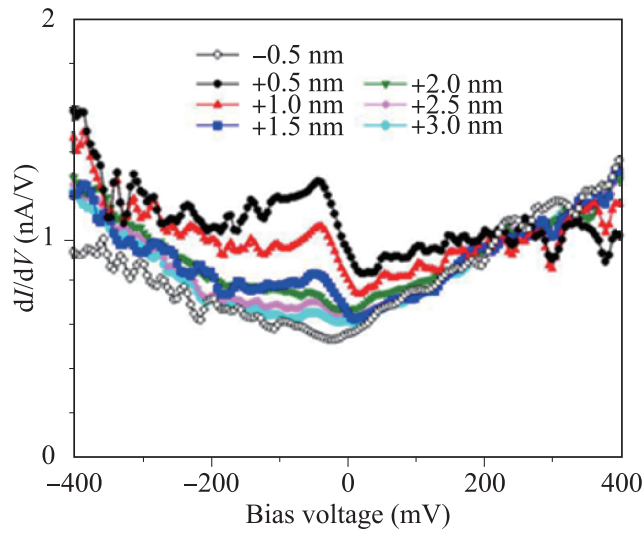

(d) Armchair edge (HOPG)

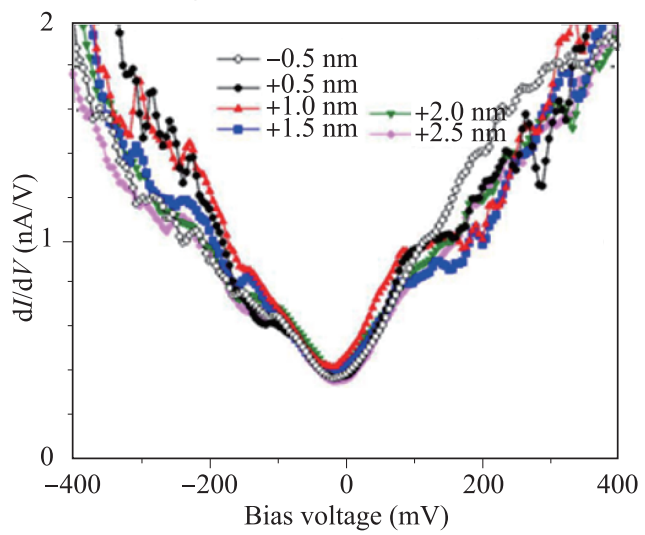

Fig. 17 (a, b) $\mathrm{d} I / \mathrm{d} V$ curve measured near zigzag edges at the surface of ZYX and HOPG. (c, d) $\mathrm{d} I / \mathrm{d} V$ curve measured near armchair edges at the surface of ZYX and HOPG. Reproduced from Ref. [83].

zigzag edge state could be clearly observed in the STS measurements, in accordance with the previous results. Moreover, a $2.3 \mathrm{~nm}$ wide zigzag-dominant GNR exhibited a smaller band gap than a $2.9 \mathrm{~nm}$ wide AGNR, another signature of zigzag edge states, as materials with zigzag edges are predicted to be metallic by tight-binding calculations.

\subsubsection{Energy gaps}

Theorists have widely studied the dependence of the energy gap on width and edge orientation, but the interplay between the precise width and edge structure in a GNR remains a rich area of experimental research [80]. The atomic resolution of STM paves the way for precise engineering of the width and edge structure of GNRs. In their pioneering work, Tapasztó et al. developed an STM-based lithography method with true nanometer precision [41]. As the differential conductance $(\mathrm{d} I / \mathrm{d} V)$ quantity can be considered to be proportional to the LDOS, the value of the energy band gap is given by the distance between the first pair of van Hove singularities [85]. These STM and STS measurements showed an energy gap of $0.18 \mathrm{eV}$ for $10 \mathrm{~nm}$ wide AGNRs. Further downscaling to $2.5 \mathrm{~nm}$ achieved an energy gap of up to $0.5 \mathrm{eV}$, in accordance with tight-binding theoretical calculations, where a gap of $1.2 \mathrm{eV} \mathrm{nm} / \mathrm{W}$ was introduced owing to the spatial confinement [80].

To verify the width and edge orientation dependence of energy gaps experimentally, the same research group analyzed the band gaps of samples with precise edge orientations utilizing STM and STS measurements [86]. The measured band gaps as a function of width are plotted in Fig. 18 for AGNRs and ZGNRs. For AGNRs, the band gap is inversely proportional to the width and several AGNRs with no detectable band gap have been observed, which might correspond to the $3 n+2$ class reported in tight-binding calculations. Strikingly, a different phenomenon is observed in ZGNRs. An electronic band gap of $200-300 \mathrm{meV}$ is detected for ZGNRs with widths narrower than $7 \mathrm{~nm}$, which can be attributed to interaction-induced spin ordering along the zigzag edges [80, 87, 88].

Interestingly, in contrast to density functional theory (DFT) calculations, a semiconductor to metal transition is revealed in ZGNRs wider than $8 \mathrm{~nm}$. The discrepancy between theoretical studies and experimental re- 

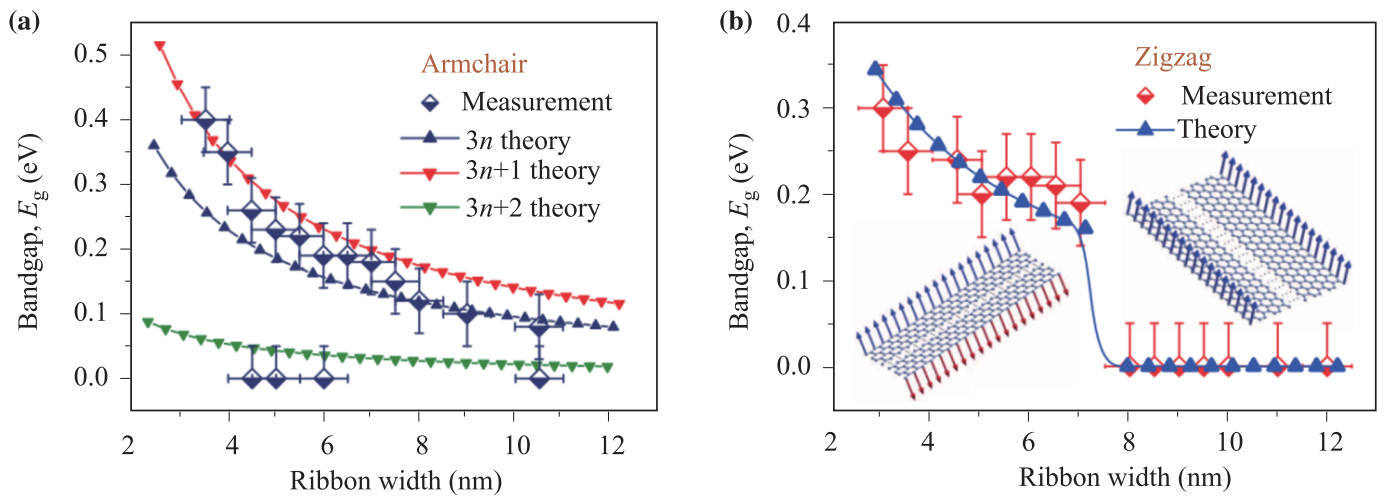

Fig. 18 (a) Bandgap measured by STS of AGNRs. (b) Bandgap measured by STS of ZGNRs. Reproduced from Ref. [86].

sults might be due to the zero temperature and lack of doping in DFT calculations, while the experimental data for ZGNRs are acquired at room temperature and finite doping. The aforementioned experimental results from STM and STS measurements indicate that both the edge configuration and width play important roles in tailoring the band gaps of GNRs. By specifically controlling the edge structure and width of GNRs, it will be possible to design GNRs with predetermined characteristics.

\section{Electron transport in graphene nanoribbons}

Electronic transport in GNRs demonstrates a number of intriguing phenomena, such as zero-conductance Fano resonances [89], valley filtering [90, 91], half metallic conduction in ZGNRs [49], the spin Hall effect [92], and a perfectly conducting channel [93]. Theoretically, the electronic transport properties of GNRs, both with rough and regular edges, have been discussed numerically. Electron scattering is described by the scattering matrix [94].

\subsection{Transport in graphene nanoribbons with different edges}

The electronic transport properties of GNRs depend greatly on their edges, which are either rough or smooth [90, 95-97]. Carrier transport through GNRs with rough edges is dominated by edge defects and can be described as variable hopping between localized states. In this case, a transport gap can be detected near the Dirac point. Interesting transport properties are predicted to exist for GNRs with perfect edges $[7,80]$. The transport theory for such GNRs can be roughly divided into several mechanisms, including Coulomb blockade and charge impurities $[95,96]$. Moreover, owing to the limitations of fabrication processes, unity between experimental research and theoretical work remains to be achieved.

\subsubsection{Transport in graphene nanoribbons with rough edges}

Han et al. reported that lithographically patterned GNRs have a transport gap that depends on the ribbon width [7]. By systematically measuring a series of ribbon devices with various widths, they found that the energy band gap scales quantitatively and inversely with the ribbon width. Remarkably, when the width of the nanoribbon is reduced to $15 \mathrm{~nm}$, a band gap as large as $200 \mathrm{meV}$ can be achieved. No signature of crystallographic directional dependence is observed, but randomly scattered values around the average $E_{\mathrm{g}}$ value corresponding to the width are observed. This phenomenon can be attributed to the disordered edge structure, as the ribbon fabrication process in their work is less likely to provide atomic control of the edge structure of GNRs.

Owing to the harsh etching conditions in the lithographic method, the edges of the obtained GNRs are rough, which could lead to the localization of charges. The edge roughness can be modeled using two regimes: weak disorder and strong disorder. Weak disorder can be considered as geometric fluctuation of the ribbon width, whereas strong disorder corresponds to small relative variation of the ribbon width, which occurs when some atoms are removed or replaced by other atoms in the fabrication process. In a theoretical study, Sols et al. attributed the transport gap observed in rough-edge GNRs to a Coulomb blockade [96]. GNRs with disordered edges, especially strong interface disorder, can be considered to form necks and dots along the ribbon, and a Coulomb blockade exists when charge transfer occurs between islands (Fig. 19). The effective charging energy of these junctions, renormalized by the charge fluctuation at the junctions, can be explained as the gap observed in the transport measurement of random-edge GNRs. In 2008, Ponomarenko et al. investigated the transport properties of GQDs experimentally and ob- 
served a Coulomb blockade of charge transfer between the dots, demonstrated by a Coulomb diamond in the conductance measurement [12].

An alternative explanation of the experimentally observed transport gap in GNRs was proposed in a model of Anderson localization [98]. In this work, Evaldsson et al. showed that a strong enhancement of LDOS exists near defects at the edges, which can be determined experimentally by utilizing STM/STS. Martin and Blanter attributed transport in disordered GNRs to effective one-dimensional hopping between segments with distinct band structures, as edge disorder leads to segmentation of the wavefunctions into blocks with lengths on the order of the GNR width using the tight-binding method [99]. Briefly, the transport properties of GNRs with rough edges are described by a combined physical picture involving charge impurities caused by the substrate, Coulomb blockage, and Anderson localization $[95,96,98]$.

\subsubsection{Transport in graphene nanoribbons with regular edges}

The transport properties of GNRs with regular edges are apparently different from those of GNRs with rough edges. Theorists have investigated the transport properties of AGNRs and ZGNRs in the universality class

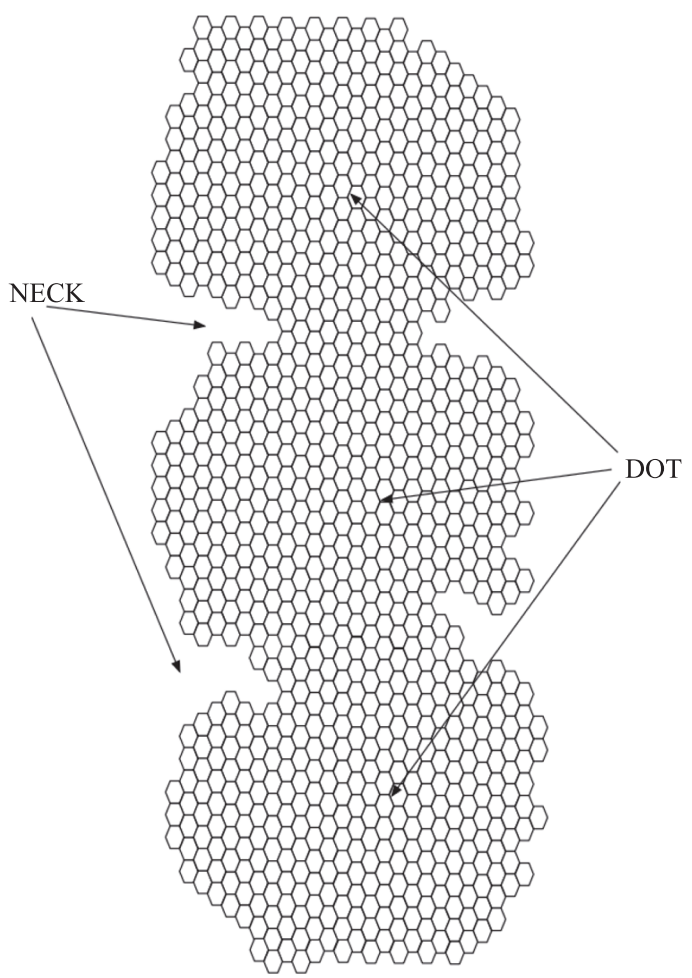

Fig. 19 Illustration of GNRs with a disordered edge, Coulomb blockade takes place when the charge moves from dot to dot. Reproduced from Ref. [96]. based on symmetry considerations $[90,91,97,100]$. Unfortunately, experimental investigations on this topic are extremely rare, partially owing to the difficulties in obtaining GNRs with well-defined edge structures.

\subsubsection{Universality class of armchair and zigzag edges}

Wakabayashi et al. investigated the transport properties of AGNRs using a tight-binding model [97]. The transport properties of AGNRs can be classified into two regimes: a low-energy single-channel transport regime and a multichannel energy regime. They observed a nearly perfect single conduction channel, even in disordered AGNRs with long-range impurities (LRIs) owing to the disappearance of the backward-scattering matrix elements in the lowest order.

As shown in Fig. 20, the average conductance of AGNRs with LRIs in the single-channel regime is nearly equal to 1, whereas for short-range impurities (SRIs), the conductance decays exponentially, even in the singlechannel regime. In comparison with AGNRs, ZGNRs have a perfectly conducting channel in the disordered system with LRIs owing to the peculiar edge state [101].

As seen in Fig. 21, the impurity range is crucial for the transport properties of ZGNRs. For SRIs [Fig. 21(b)], impurity scattering connects the two valleys, called intervalley scattering, whereas LRIs restrict the scattering process and lead to intravalley scattering. For LRIs [Fig. 21(a)], the average conductance gradually decreases with increasing length $L$, and notably, the average conductance converges to 1 , indicating the perfectly conducting channel in disordered ZGNRs in LRIs. Intervalley scattering in the SRIs causes back scattering, and the average conductance decays exponentially without developing a perfectly conducting channel.

A symmetry consideration for regular-edge GNRs with different impurities has been considered to identify the universality classes in ZGNRs and AGNRs. The universality classes describe transport properties according to random matrix theory based on time-reversal symmetry (TRS) and spin-rotation symmetry and can be classified into three categories: orthogonal, unitary, and symplectic [93]. The orthogonal class has both TRS and spinrotation symmetry, whereas the unitary class is characterized by the absence of TRS. The symplectic class features TRS without spin-rotation symmetry.

In the GNR systems, the range of the impurity potential is related to the presence or absence of TRS. ZGNRs and AGNRs differ from each other in terms of the boundary conditions. Two well-separated valleys exist in $\mathrm{k}$ space for ZGNRs, whereas two valleys merge into a single valley at $k=0$ for AGNRs. In ZGNRs, SRIs yield intervalley scattering that restore TRS for both valleys by incorporating a complete set of pairs of time-resolved modes. In contrast, LRIs in ZGNRs cause intravalley 

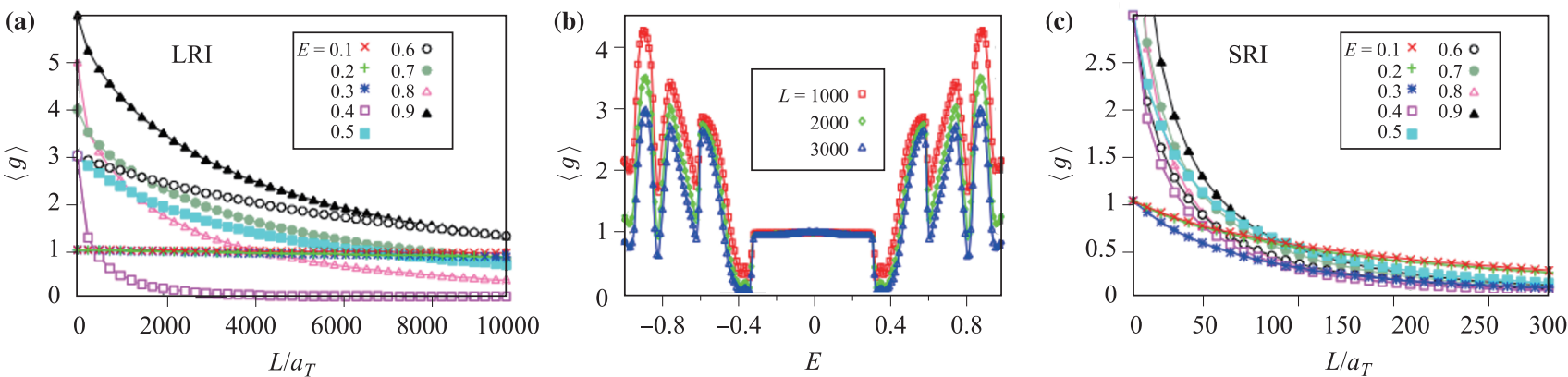

Fig. 20 (a, c) Averaged conductance of AGNRs as a function of length in the presence of LRI and SRI, respectively. (b) The energy dependence of averaged conductance of armchair GNRs in the presence of LRI. Reproduced from Ref. [97].
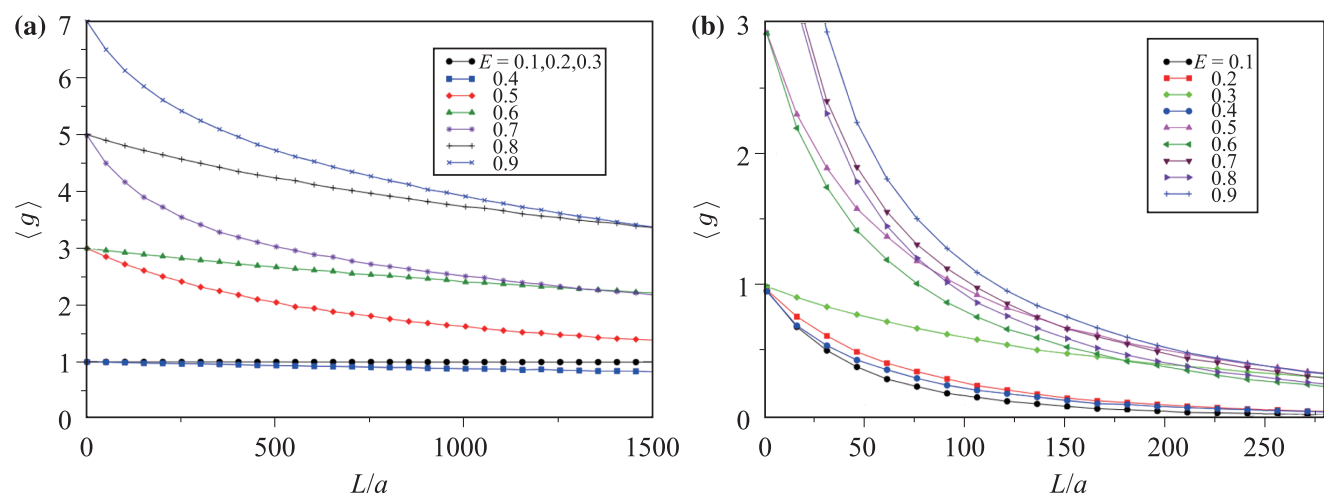

Fig. 21 (a, b) Averaged conductance of ZGNRs as a function of length in LRIs and SRIs, respectively. Reproduced from Ref. [101].

scattering, which might destroy TRS. Thus, ZGNRs with LRIs without TRS belong to the unitary class, whereas ZGNRs with SRIs with well-protected TRS belong to the orthogonal class. In the case of AGNRs, owing to merging of two valleys at $k=0$, TRS is conserved irrespective of the impurity potential range. Therefore, AGNRs belong to the orthogonal class and do not provide a perfectly conducting channel like that of ZGNRs. It is quite interesting that the universality class is influenced by the edge termination of GNRs. Moreover, with the rapid progress of experiments confirming the edge orientation at the atomic level, it is of great interest to investigate the transport properties of GNRs with well-defined regular edges experimentally [34].

\subsubsection{Unique transport properties of zigzag graphene nanoribbons}

Owing to the existence of edge states, ZGNRs are characterized by a number of interesting transport properties, such as a half-metallic state and valley filtering $[49,90,91]$. Louie and co-workers predicted halfmetallicity in ZGNRs with an in-plane homogeneous electric field based on the ab initio pseudopotential density function method within the local spin density approximation (LSDA) [49]. By considering the effect of spin and electric field, energy level shifts of spin-ordered edge states were observe to be induced by an applied electric field. They labeled the gap-opening states as $\alpha$-spin and the gap-closing states as $\beta$-spin. The halfmetallic property of ZGNRs means that the metallic nature of electrons with $\beta$-spin coexists with the insulating nature of electrons with $\alpha$-spin, as shown in Fig. 22. Moreover, they confirmed the robustness of the predicted half-metallicity with different kinds of defects, such as dangling bonds, vacancies, and Stone-Wales defects, at a defect concentration of $6 \%-12 \%$ per edge.

In addition to spin and charge degrees of freedom in ZGNRs, the peculiar band structure makes it possible to utilize the valley degree of freedom in ZGNRs. Rycerz et $a l$. proposed that a valley filter and valley valve could be obtained in graphene based on a ballistic point contact with zigzag edges [90]. A schematic diagram of the valley filter is shown in Fig. 23, in which an electron in the first valley (filled circle) is transmitted, while an electron in the second valley (open circle) is reflected. Wakabayashi and Aoki proposed the polarization of the two valleys as the origin of the current blocking effect [102]. The current blocking effect showed that the current is almost blocked by the potential barrier when the incident energy is in the range of $[0, \Delta]$, while the barrier potential height, $V_{o}$, is in the range of $[\Delta, 2 \Delta]$, where $\Delta$ represents the 
spacing of the low-lying modes.

\subsection{Quantum Hall effect of two-terminal graphene nanoribbon devices}

An interesting aspect of graphene is the ability of charge carriers to travel ballistically over hundreds of nanometers. Theoretically, ZGNRs can be a platform to study the quantum spin Hall effect [92], quantum valley Hall effect [104, 105], and quantum anomalous Hall effect [106], which can be tuned by Rashba spin-orbit coupling, exchange fields, and interlayer potentials for multilayer GNRs. However, experimental observation of the quantum Hall effect is very challenging as the GNR system is diffusive, disordered, and lacks uniform dop-

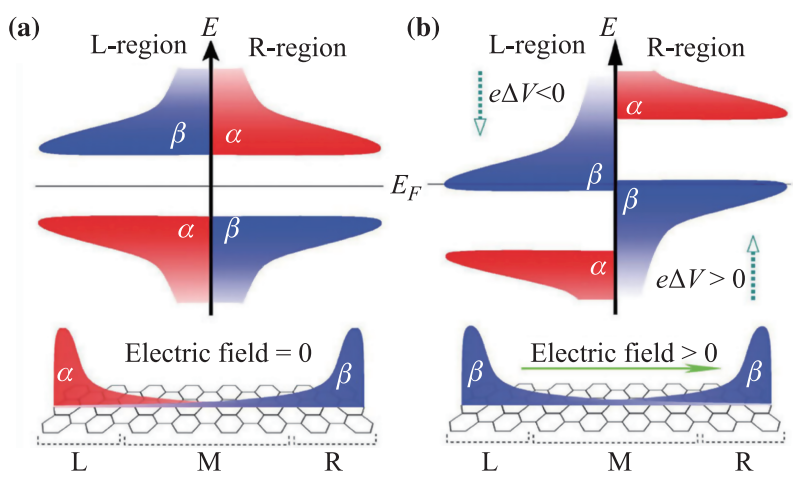

Fig. 22 (a) Oppositely oriented spin states of ZGNRs. (b) Applied electric fields induce energy-level shifts of opposite signs for the spatially separated spin-ordered edge states. Reproduced from Ref. [49].
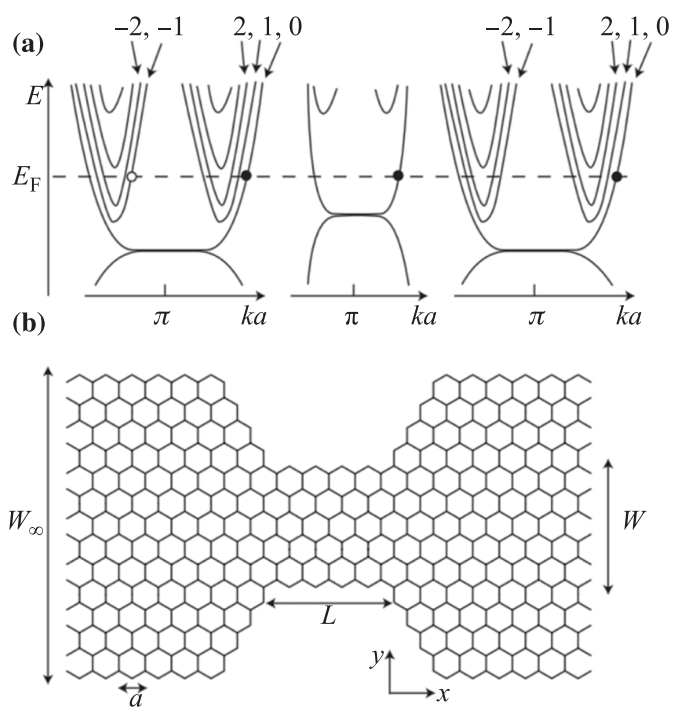

Fig. 23 (a) Dispersion relations in the wide and narrow regions. An electron in the first valley (filled circle) is transmitted while an electron in the second valley is reflected (open circle). (b) Schematic diagram of valley filter. Reported from Ref. [90]. ing owing to strong interaction with the substrate [103]. One way to circumvent this problem is to fabricate suspended GNRs to avoid the influence of the substrate and prepare a constricted length comparable to or shorter than the width to decrease the edge disorder. Tombros et al. reported the observation of quantized conductance for a suspended graphene nanoconstriction in the ballistic regime [107]. A pronounced feature at $0.6 G_{0}$ $\left(G_{0}=2 e^{2} / h\right)$ occurred at magnetic fields as low as $\sim 0.2$ $\mathrm{T}$, possibly caused by electron-electron interactions, indicating the high quality of the suspended GNR samples prepared by a current annealing method. Crossover from the Coulomb blockade, which originated from geometric confinement caused by edge disorder, to the quantum Hall effect, which is due to magnetic confinement in suspended GNRs, was observed by Ki and Morpurgo in 2012 using a similar current annealing method [108]. As the length of the GNRs presented by Ki and Morpurgo is much longer than that of the GNRs fabricated by Tombros et al., the edge scattering is much severe, resulting in electron localization and prevention of ballistic transport. Though the fabrication of high-quality suspended GNRs prepared by current annealing methods paves the way for exploring the properties of confined Dirac electrons in different transport regimes, the limitation of this method is uncertainty in the actual width of the GNRs, as brittleness is a known problem of suspended GNRs. Therefore, the relationship between the subband energy spacing and the ribbon width cannot be obtained accurately in transport experiments for GNRs produced using current annealing. As boron nitride has proved a high-quality substrate for graphene, the problem of determining the actual width of GNRs might be solved by patterning high-quality GNRs on boron nitride substrates [36, 70, 109].

\section{Conclusions and outlook}

Various methods for fabricating GNRs have been demonstrated using top-down approaches as well as bottom-up approaches. In addition, the electronic and transport properties of GNRs have been discussed in detail, both theoretically and experimentally. Though the band gap of GNRs can be opened by the quantum confinement effect, there are still limitations for application of GNRs in logical devices, which also require high carrier mobility. However, GNRs, especially those with zigzag terminations, show great potential in spintronics and valleytronics owing to their unique electronic states. To explore spin and valley transport in ZGNRs, high-quality GNRs with well-defined edges and ultra-high carrier mobilities are essential. We anticipate that the experimental realization of high-quality ZGNRs on boron nitride will make 
it possible to exploit the valley degree of freedom, in addition to the spin and charge degrees of freedom in the ZGNRs, as an information carrier in carbon electronics.

Acknowledgements This work was supported by the National Natural Science Foundation of China (NSFC, Grant Nos. 61325021, 11574361, and 61390503), the National Basic Research Program of China (973 Program, Grant Nos. 2013CB934500 and 2013CBA01602), and the Key Research Program of Frontier Sciences (Grant No. QYZDB-SSW-SLH004).

Open Access This article is distributed under the terms of the Creative Commons Attribution License which permits any use, distribution, and reproduction in any medium, provided the original author(s) and the source are credited.

\section{References}

1. K. S. Novoselov, A. K. Geim, S. V. Morozov, D. Jiang, Y. Zhang, S. V. Dubonos, I. V. Grigorieva, and A. A. Firsov, Electric field effect in atomically thin carbon films, Science 306(5696), 666 (2004)

2. K. S. Novoselov, A. K. Geim, S. V. Morozov, D. Jiang, M. I. Katsnelson, I. V. Grigorieva, S. V. Dubonos, and A. A. Firsov, Two-dimensional gas of massless Dirac fermions in graphene, Nature 438(7065), 197 (2005)

3. M. I. Katsnelson, K. S. Novoselov, and A. K. Geim, Chiral tunnelling and the Klein paradox in graphene, Nat. Phys. 2(9), 620 (2006)

4. J. Baringhaus, M. Ruan, F. Edler, A. Tejeda, M. Sicot, A. Taleb-Ibrahimi, A. P. Li, Z. Jiang, E. H. Conrad, C. Berger, C. Tegenkamp, and W. A. de Heer, Exceptional ballistic transport in epitaxial graphene nanoribbons, Nature 506(7488), 349 (2014)

5. C. Berger, Z. Song, X. Li, X. Wu, N. Brown, C. Naud, D. Mayou, T. Li, J. Hass, A. N. Marchenkov, E. H. Conrad, P. N. First, and W. A. de Heer, Electronic confinement and coherence in patterned epitaxial graphene, Science 312(5777), 1191 (2006)

6. M. Orlita, C. Faugeras, P. Plochocka, P. Neugebauer, G. Martinez, D. K. Maude, A. L. Barra, M. Sprinkle, C. Berger, W. A. de Heer, and M. Potemski, Approaching the Dirac point in high-mobility multilayer epitaxial graphene, Phys. Rev. Lett. 101(26), 267601 (2008)

7. M. Y. Han, B. Ozyilmaz, Y. Zhang, and P. Kim, Energy band-gap engineering of graphene nanoribbons, Phys. Rev. Lett. 98(20), 206805 (2007)

8. L. Jiao, X. Wang, G. Diankov, H. Wang, and H. Dai, Facile synthesis of high-quality graphene nanoribbons, Nat. Nanotechnol. 5(5), 321 (2010)

9. D. V. Kosynkin, A. L. Higginbotham, A. Sinitskii, J. R. Lomeda, A. Dimiev, B. K. Price, and J. M. Tour, Longitudinal unzipping of carbon nanotubes to form graphene nanoribbons, Nature 458(7240), 872 (2009)
10. J. W. Bai, X. Zhong, S. Jiang, Y. Huang, and X. F. Duan, Graphene nanomesh, Nat. Nanotechnol. 5(3), 190 (2010)

11. X. Liang, Y. S. Jung, S. Wu, A. Ismach, D. L. Olynick, S. Cabrini, and J. Bokor, Formation of bandgap and subbands in graphene nanomeshes with sub-10 nm ribbon width fabricated via nanoimprint lithography, Nano Lett. 10(7), 2454 (2010)

12. L. A. Ponomarenko, F. Schedin, M. I. Katsnelson, R. Yang, E. W. Hill, K. S. Novoselov, and A. K. Geim, Chaotic Dirac billiard in graphene quantum dots, Science 320(5874), 356 (2008)

13. B. Song, G. F. Schneider, Q. Xu, G. Pandraud, C. Dekker, and H. Zandbergen, Atomic-scale electronbeam sculpting of near-defect-free graphene nanostructures, Nano Lett. 11(6), 2247 (2011)

14. A. Sinitskii and J. M. Tour, Patterning graphene through the self-assembled templates: Toward periodic two-dimensional graphene nanostructures with semiconductor properties, J. Am. Chem. Soc. 132(42), 14730 (2010)

15. L. Liu, Y. Zhang, W. Wang, C. Gu, X. Bai, and E. Wang, Nanosphere lithography for the fabrication of ultranarrow graphene nanoribbons and on-chip bandgap tuning of graphene, Adv. Mater. 23(10), 1246 (2011)

16. M. Kim, N. S. Safron, E. Han, M. S. Arnold, and P. Gopalan, Fabrication and characterization of largearea, semiconducting nanoperforated graphene materials, Nano Lett. 10(4), 1125 (2010)

17. J. G. Son, M. Son, K. J. Moon, B. H. Lee, J. M. Myoung, M. S. Strano, M. H. Ham, and C. A. Ross, Sub$10 \mathrm{~nm}$ graphene nanoribbon array field-effect transistors fabricated by block copolymer lithography, Adv. Mater. 25(34), 4723 (2013)

18. A. Sinitskii and J. M. Tour, Patterning graphene nanoribbons using copper oxide nanowires, Appl. Phys. Lett. 100(10), 103106 (2012)

19. L. Liao, J. Bai, R. Cheng, Y. C. Lin, S. Jiang, Y. Huang, and $\mathrm{X}$. Duan, Top-gated graphene nanoribbon transistors with ultrathin high- $k$ dielectrics, Nano Lett. 10(5), 1917 (2010)

20. L. Liao, J. Bai, Y. C. Lin, Y. Qu, Y. Huang, and $\mathrm{X}$. Duan, High-performance top-gated graphenenanoribbon transistors using zirconium oxide nanowires as high-dielectric-constant gate dielectrics, Adv. Mater. 22(17), 1941 (2010)

21. L. Liao, Y. C. Lin, M. Bao, R. Cheng, J. Bai, Y. Liu, Y. Qu, K. L. Wang, Y. Huang, and X. Duan, High-speed graphene transistors with a self-aligned nanowire gate, Nature 467(7313), 305 (2010)

22. W. Xu, H. K. Seo, S. Y. Min, H. Cho, T. S. Lim, C. Y. Oh, Y. Lee, and T. W. Lee, Rapid fabrication of designable large-scale aligned graphene nanoribbons by electro-hydrodynamic nanowire lithography, $A d v$. Mater. 26(21), 3459 (2014) 
23. S. Park, D. H. Lee, J. Xu, B. Kim, S. W. Hong, U. Jeong, T. Xu, and T. P. Russell, Macroscopic 10-terabit-persquare-inch arrays from block copolymers with lateral order, Science 323(5917), 1030 (2009)

24. Z. Huo, C. K. Tsung, W. Huang, M. Fardy, R. Yan, X. Zhang, Y. Li, and P. Yang, Self-organized ultrathin oxide nanocrystals, Nano Lett. 9(3), 1260 (2009)

25. J. D. Holmes, K. P. Johnston, R. Christopher Doty, and B. A. Korgel, Control of thickness and orientation of solution-grown silicon nanowires, Science 287(5457), 1471 (2000)

26. C. Wang, Y. J. Hu, C. M. Lieber, and S. H. Sun, Ultrathin $\mathrm{Au}$ nanowires and their transport properties, $J$. Am. Chem. Soc. 130(28), 8902 (2008)

27. X. Lu, M. S. Yavuz, H. Y. Tuan, B. A. Korgel, and Y. Xia, Ultrathin gold nanowires can be obtained by reducing polymeric strands of oleylamine-AuCl complexes formed via aurophilic interaction, J. Am. Chem. Soc. 130(28), 8900 (2008)

28. J. W. Bai, X. F. Duan, and Y. Huang, Rational fabrication of graphene nanoribbons using a nanowire etch mask, Nano Lett. 9(5), 2083 (2009)

29. T. O. Wehling, S. Yuan, A. I. Lichtenstein, A. K. Geim, and M. I. Katsnelson, Resonant scattering by realistic impurities in graphene, Phys. Rev. Lett. 105(5), 056802 (2010)

30. L. C. Campos, V. R. Manfrinato, J. D. SanchezYamagishi, J. Kong, and P. Jarillo-Herrero, Anisotropic etching and nanoribbon formation in single-layer graphene, Nano Lett. 9(7), 2600 (2009)

31. S. S. Datta, D. R. Strachan, S. M. Khamis, and A. T. C. Johnson, Crystallographic etching of few-layer graphene, Nano Lett. 8(7), 1912 (2008)

32. L. Ci, Z. Xu, L. Wang, W. Gao, F. Ding, K. F. Kelly, B. I. Yakobson, and P. M. Ajayan, Controlled nanocutting of graphene, Nano Res. 1(2), 116 (2008)

33. L. Ci, L. Song, D. Jariwala, A. L. ElÃas, W. Gao, M. Terrones, and P. M. Ajayan, Graphene shape control by multistage cutting and transfer, Adv. Mater. 21(44), 4487 (2009)

34. R. Yang, L. Zhang, Y. Wang, Z. Shi, D. Shi, H. Gao, E. Wang, and G. Zhang, An anisotropic etching effect in the graphene Basal plane, Adv. Mater. 22(36), 4014 (2010)

35. Z. Shi, R. Yang, L. Zhang, Y. Wang, D. Liu, D. Shi, E. Wang, and G. Zhang, Patterning graphene with zigzag edges by self-aligned anisotropic etching, Adv. Mater. 23(27), 3061 (2011)

36. G. Wang, S. Wu, T. Zhang, P. Chen, X. Lu, S. Wang, D. Wang, K. Watanabe, T. Taniguchi, D. Shi, R. Yang, and G. Zhang, Patterning monolayer graphene with zigzag edges on hexagonal boron nitride by anisotropic etching, Appl. Phys. Lett. 109(5), 053101 (2016)

37. L. Weng, L. Zhang, Y. P. Chen, and L. P. Rokhinson, Atomic force microscope local oxidation nanolithography of graphene, Appl. Phys. Lett. 93(9), 093107 (2008)
38. G. Lu, X. Zhou, H. Li, Z. Yin, B. Li, L. Huang, F. Boey, and H. Zhang, Nanolithography of single-layer graphene oxide films by atomic force microscopy, Langmuir 26(9), 6164 (2010)

39. K. Zhang, Q. Fu, N. Pan, X. Yu, J. Liu, Y. Luo, X. Wang, J. Yang, and J. Hou, Direct writing of electronic devices on graphene oxide by catalytic scanning probe lithography, Nat. Commun. 3, 1194 (2012)

40. S. Masubuchi, M. Ono, K. Yoshida, K. Hirakawa, and T. Machida, Fabrication of graphene nanoribbon by local anodic oxidation lithography using atomic force microscope, Appl. Phys. Lett. 94(8), 082107 (2009)

41. L. Tapasztó, G. Dobrik, P. Lambin, and L. P. Biró, Tailoring the atomic structure of graphene nanoribbons by scanning tunnelling microscope lithography, Nat. Nanotechnol. 3(7), 397 (2008)

42. M. D. Fischbein and M. Drndić, Electron beam nanosculpting of suspended graphene sheets, Appl. Phys. Lett. 93(11), 113107 (2008)

43. D. C. Bell, M. C. Lemme, L. A. Stern, J. R. Williams, and C. M. Marcus, Precision cutting and patterning of graphene with helium ions, Nanotechnology 20(45), 455301 (2009)

44. D. Winston, V. R. Manfrinato, S. M. Nicaise, L. L. Cheong, H. Duan, D. Ferranti, J. Marshman, S. McVey, L. Stern, J. Notte, and K. K. Berggren, Neon ion beam lithography (NIBL), Nano Lett. 11(10), 4343 (2011)

45. A. Lerf, H. He, M. Forster, and J. Klinowski, Structure of graphite oxide revisited, J. Phys. Chem. B 102(23), 4477 (1998)

46. L. Jiao, L. Zhang, L. Ding, J. Liu, and H. Dai, Aligned graphene nanoribbons and crossbars from unzipped carbon nanotubes, Nano Res. 3(6), 387 (2010)

47. L. Xie, H. Wang, C. Jin, X. Wang, L. Jiao, K. Suenaga, and H. Dai, Graphene nanoribbons from unzipped carbon nanotubes: Atomic structures, Raman spectroscopy, and electrical properties, J. Am. Chem. Soc. 133(27), 10394 (2011)

48. X. Wang, Y. Ouyang, L. Jiao, H. Wang, L. Xie, J. Wu, J. Guo, and H. Dai, Graphene nanoribbons with smooth edges behave as quantum wires, Nat. Nanotechnol. 6(9), 563 (2011)

49. Y. W. Son, M. L. Cohen, and S. G. Louie, Half-metallic graphene nanoribbons, Nature 444(7117), 347 (2006)

50. B. Trauzettel, D. V. Bulaev, D. Loss, and G. Burkard, Spin qubits in graphene quantum dots, Nat. Phys. 3(3), 192 (2007)

51. X. Yang, X. Dou, A. Rouhanipour, L. Zhi, H. J. Rader, and K. Mullen, Two-dimensional graphene nanoribbons, J. Am. Chem. Soc. 130(13), 4216 (2008)

52. A. Narita, X. Feng, Y. Hernandez, S. A. Jensen, M. Bonn, H. Yang, I. A. Verzhbitskiy, C. Casiraghi, M. R. Hansen, A. H. Koch, G. Fytas, O. Ivasenko, B. Li, K. S. Mali, T. Balandina, S. Mahesh, S. De Feyter, and K. Mullen, Synthesis of structurally well-defined and 
liquid-phase-processable graphene nanoribbons, Nat. Chem. 6(2), 126 (2013)

53. J. Cai, P. Ruffieux, R. Jaafar, M. Bieri, T. Braun, S. Blankenburg, M. Muoth, A. P. Seitsonen, M. Saleh, X. Feng, K. Mullen, and R. Fasel, Atomically precise bottom-up fabrication of graphene nanoribbons, Nature 466(7305), 470 (2010)

54. P. Ruffieux, S. Wang, B. Yang, C. Sanchez-Sanchez, J. Liu, T. Dienel, L. Talirz, P. Shinde, C. A. Pignedoli, D. Passerone, T. Dumslaff, X. Feng, K. Mullen, and R. Fasel, On-surface synthesis of graphene nanoribbons with zigzag edge topology, Nature 531(7595), 489 (2016)

55. S. Blankenburg, J. Cai, P. Ruffieux, R. Jaafar, D. Passerone, X. Feng, K. Müllen, R. Fasel, and C. A. Pignedoli, Intraribbon heterojunction formation in ultranarrow graphene nanoribbons, ACS Nano 6(3), 2020 (2012)

56. J. Cai, C. A. Pignedoli, L. Talirz, P. Ruffieux, H. Sode, L. Liang, V. Meunier, R. Berger, R. Li, X. Feng, K. Mullen, and R. Fasel, Graphene nanoribbon heterojunctions, Nat. Nanotechnol. 9(11), 896 (2014)

57. Y. C. Chen, T. Cao, C. Chen, Z. Pedramrazi, D. Haberer, D. G. de Oteyza, F. R. Fischer, S. G. Louie, and M. F. Crommie, Molecular bandgap engineering of bottom-up synthesized graphene nanoribbon heterojunctions, Nat. Nanotechnol. 10(2), 156 (2015)

58. P. Ruffieux, J. Cai, N. C. Plumb, L. Patthey, D. Prezzi, A. Ferretti, E. Molinari, X. Feng, K. Müllen, C. A. Pignedoli, and R. Fasel, Electronic structure of atomically precise graphene nanoribbons, ACS Nano 6, 6930 (2012)

59. Y.C. Chen, D. G. de Oteyza, Z. Pedramrazi, C. Chen, F. R. Fischer, and M. F. Crommie, Tuning the band gap of graphene nanoribbons synthesized from molecular precursors, ACS Nano 7(7), 6123 (2013)

60. T. H. Vo, M. Shekhirev, D. A. Kunkel, M. D. Morton, E. Berglund, L. Kong, P. M. Wilson, P. A. Dowben, A. Enders, and A. Sinitskii, Large-scale solution synthesis of narrow graphene nanoribbons, Nat. Commun. 5, 3189 (2014)

61. X. Li, W. Cai, J. An, S. Kim, J. Nah, D. Yang, R. Piner, A. Velamakanni, I. Jung, E. Tutuc, S. K. Banerjee, L. Colombo, and R. S. Ruoff, Large-area synthesis of high-quality and uniform graphene films on copper foils, Science 324(5932), 1312 (2009)

62. M. Sprinkle, M. Ruan, Y. Hu, J. Hankinson, M. RubioRoy, B. Zhang, X. Wu, C. Berger, and W. A. de Heer, Scalable templated growth of graphene nanoribbons on SiC, Nat. Nanotechnol. 5(10), 727 (2010)

63. Q. Huang, J. J. Kim, G. Ali, and S. O. Cho, Widthtunable graphene nanoribbons on a $\mathrm{SiC}$ substrate with a controlled step height, Adv. Mater. 25(8), 1144 (2013)

64. M. S. Nevius, F. Wang, C. Mathieu, N. Barrett, A. Sala, T. O. Mentes, A. Locatelli, and E. H. Conrad, The bottom-up growth of edge specific graphene nanoribbons, Nano Lett. 14(11), 6080 (2014)
65. K. Hayashi, S. Sato, M. Ikeda, C. Kaneta, and N. Yokoyama, Selective graphene formation on Copper twin crystals, J. Am. Chem. Soc. 134(30), 12492 (2012)

66. T. Kato and R. Hatakeyama, Site- and alignmentcontrolled growth of graphene nanoribbons from nickel nanobars, Nat. Nanotechnol. 7(10), 651 (2012)

67. I. Martin-Fernandez, D. Wang, and Y. Zhang, Direct growth of graphene nanoribbons for large-scale device fabrication, Nano Lett. 12(12), 6175 (2012)

68. H. Ago, I. Tanaka, Y. Ogawa, R. M. Yunus, M. Tsuji, and H. Hibino, Lattice-oriented catalytic growth of graphene nanoribbons on heteroepitaxial nickel films, ACS Nano 7(12), 10825 (2013)

69. R. M. Jacobberger, B. Kiraly, M. Fortin-Deschenes, P. L. Levesque, K. M. McElhinny, G. J. Brady, R. Rojas Delgado, S. Singha Roy, A. Mannix, M. G. Lagally, P. G. Evans, P. Desjardins, R. Martel, M. C. Hersam, N. P. Guisinger, and M. S. Arnold, Direct oriented growth of armchair graphene nanoribbons on germanium, Nat. Commun. 6, 8006 (2015)

70. X. Lu, W. Yang, S. Wang, S. Wu, P. Chen, J. Zhang, J. Zhao, J. Meng, G. Xie, D. Wang, G. Wang, T. T. Zhang, K. Watanabe, T. Taniguchi, R. Yang, D. Shi, and G. Zhang, Graphene nanoribbons epitaxy on boron nitride, Appl. Phys. Lett. 108(11), 113103 (2016)

71. M. Fujita, K. Wakabayashi, K. Nakada, and K. Kusakabe, Peculiar localized state at zigzag graphite edge, $J$. Phys. Soc. Jpn. 65(7), 1920 (1996)

72. K. Nakada, M. Fujita, G. Dresselhaus, and M. S. Dresselhaus, Edge state in graphene ribbons: Nanometer size effect and edge shape dependence, Phys. Rev. B 54(24), 17954 (1996)

73. L. Brey and H. A. Fertig, Electronic states of graphene nanoribbons studied with the Dirac equation, Phys. Rev. B 73(23), 235411 (2006)

74. M. Wimmer, A. R. Akhmerov, and F. Guinea, Robustness of edge states in graphene quantum dots, Phys. Rev. B 82(4), 045409 (2010)

75. K. Wakabayashi, M. Fujita, H. Ajiki, and M. Sigrist, Electronic and magnetic properties of nanographite ribbons, Phys. Rev. B 59(12), 8271 (1999)

76. J. Fernández-Rossier and J. J. Palacios, Magnetism in graphene nanoislands, Phys. Rev. Lett. 99(17), 177204 (2007)

77. K. Wakabayashi, S. Okada, R. Tomita, S. Fujimoto, and Y. Natsume, Edge states and flat bands of graphene nanoribbons with edge modification, J. Phys. Soc. Jpn. 79(3), 034706 (2010)

78. M. Ezawa, Peculiar width dependence of the electronic properties of carbon nanoribbons, Phys. Rev. B 73(4), 045432 (2006)

79. V. Barone, O. Hod, and G. E. Scuseria, Electronic structure and stability of semiconducting graphene nanoribbons, Nano Lett. 6(12), 2748 (2006) 
80. Y. W. Son, M. L. Cohen, and S. G. Louie, Energy gaps in graphene nanoribbons, Phys. Rev. Lett. 97(21), 216803 (2006)

81. Z. Klusek, Z. Waqar, E. A. Denisov, T. N. Kompaniets, I. V. Makarenko, A. N. Titkov, and A. S. Bhatti, Observations of local electron states on the edges of the circular pits on hydrogen-etched graphite surface by scanning tunneling spectroscopy, Appl. Surf. Sci. 161(3-4), 508 (2000)

82. Y. Kobayashi, K.i. Fukui, T. Enoki, K. Kusakabe, and Y. Kaburagi, Observation of zigzag and armchair edges of graphite using scanning tunneling microscopy and spectroscopy, Phys. Rev. B 71, 193406 (2005)

83. Y. Niimi, T. Matsui, H. Kambara, K. Tagami, M. Tsukada, and H. Fukuyama, Scanning tunneling microscopy and spectroscopy of the electronic local density of states of graphite surfaces near monoatomic step edges, Phys. Rev. B 73(8), 085421 (2006)

84. K. A. Ritter and J. W. Lyding, The influence of edge structure on the electronic properties of graphene quantum dots and nanoribbons, Nat. Mater. 8(3), 235 (2009)

85. J. G. Wildoer, L. C. Venema, A. G. Rinzler, R. E. Smalley, and C. Dekker, Electronic structure of atomically resolved carbon nanotubes, Nature 391(6662), 59 (1998)

86. G. Z. Magda, X. Jin, I. Hagymasi, P. Vancso, Z. Osvath, P. Nemes-Incze, C. Hwang, L. P. Biro, and L. Tapaszto, Room-temperature magnetic order on zigzag edges of narrow graphene nanoribbons, Nature 514(7524), 608 (2014)

87. J. Jung and A. H. MacDonald, Carrier density and magnetism in graphene zigzag nanoribbons, Phys. Rev. B $79(23), 235433$ (2009)

88. M. Golor, T. C. Lang, and S. Wessel, Quantum Monte Carlo studies of edge magnetism in chiral graphene nanoribbons, Phys. Rev. B 87(15), 155441 (2013)

89. K. Wakabayashi and M. Sigrist, Zero-conductance resonances due to flux states in nanographite ribbon junctions, Phys. Rev. Lett. 84(15), 3390 (2000)

90. A. Rycerz, J. Tworzydło, and C. W. J. Beenakker, Valley filter and valley valve in graphene, Nat. Phys. 3(3), 172 (2007)

91. A. R. Akhmerov, J. H. Bardarson, A. Rycerz, and C. W. J. Beenakker, Theory of the valley-valve effect in graphene nanoribbons, Phys. Rev. B 77(20), 205416 (2008)

92. C. L. Kane and E. J. Mele, Quantum spin Hall effect in graphene, Phys. Rev. Lett. 95(22), 226801 (2005)

93. K. Wakabayashi, Y. Takane, and M. Sigrist, Perfectly conducting channel and universality crossover in disordered graphene nanoribbons, Phys. Rev. Lett. 99(3), 036601 (2007)

94. C. W. J. Beenakker, Random-matrix theory of quantum transport, Rev. Mod. Phys. 69(3), 731 (1997)
95. S. Adam, S. Cho, M. S. Fuhrer, and S. Das Sarma, Density Inhomogeneity driven percolation metal-insulator transition and dimensional crossover in graphene nanoribbons, Phys. Rev. Lett. 101(4), 046404 (2008)

96. F. Sols, F. Guinea, and A. H. Neto, Coulomb blockade in graphene nanoribbons, Phys. Rev. Lett. 99(16), 166803 (2007)

97. M. Yamamoto, Y. Takane, and K. Wakabayashi, Nearly perfect single-channel conduction in disordered armchair nanoribbons, Phys. Rev. B 79(12), 125421 (2009)

98. M. Evaldsson, I. V. Zozoulenko, H. Xu, and T. Heinzel, Edge-disorder-induced Anderson localization and conduction gap in graphene nanoribbons, Phys. Rev. B 78(16), 161407 (2008)

99. I. Martin and Y. M. Blanter, Transport in disordered graphene nanoribbons, Phys. Rev. B 79(23), 235132 (2009)

100. H. Suzuura and T. Ando, Crossover from symplectic to orthogonal class in a two-dimensional honeycomb lattice, Phys. Rev. Lett. 89(26), 266603 (2002)

101. K. Wakabayashi, Y. Takane, and M. Sigrist, Perfectly conducting channel and universality crossover in disordered graphene nanoribbons, Phys. Rev. Lett. 99(3), 036601 (2007)

102. K. Wakabayashi and T. Aoki, Electrical conductance of zigzag nanographite ribbons with locally applied gate voltage, Int. J. Mod. Phys. B 16(32), 4897 (2002)

103. E. R. Mucciolo, A. H. Castro Neto, and C. H. Lewenkopf, Conductance quantization and transport gaps in disordered graphene nanoribbons, Phys. Rev. B 79(7), 075407 (2009)

104. Z. Qiao, X. Li, W. K. Tse, H. Jiang, Y. Yao, and Q. Niu, Topological phases in gated bilayer graphene: Effects of Rashba spin-orbit coupling and exchange field, Phys. Rev. B 87(12), 125405 (2013)

105. Z. Qiao, S. A. Yang, B. Wang, Y. Yao, and Q. Niu, Spinpolarized and valley helical edge modes in graphene nanoribbons, Phys. Rev. B 84(3), 035431 (2011)

106. Z. Qiao, W. Ren, H. Chen, L. Bellaiche, Z. Zhang, A. H. Macdonald, and Q. Niu, Quantum anomalous Hall effect in graphene proximity coupled to an antiferromagnetic insulator, Phys. Rev. Lett. 112(11), 116404 (2014)

107. N. Tombros, A. Veligura, J. Junesch, M. H. D. Guimarães, I. J. Vera-Marun, H. T. Jonkman, and B. J. van Wees, Quantized conductance of a suspended graphene nanoconstriction, Nat. Phys. 7(9), 697 (2011)

108. D. K. Ki and A. F. Morpurgo, Crossover from coulomb blockade to quantum Hall effect in suspended graphene nanoribbons, Phys. Rev. Lett. 108(26), 266601 (2012)

109. C. R. Dean, A. F. Young, I. Meric, C. Lee, L. Wang, S. Sorgenfrei, K. Watanabe, T. Taniguchi, P. Kim, K. L. Shepard, and J. Hone, Boron nitride substrates for highquality graphene electronics, Nat. Nanotechnol. 5(10), 722 (2010) 